Florida International University FIU Digital Commons

\title{
Evaluating the effectiveness of signal timing optimization based on microscopic simulation evaluation
}

Patricio Alvarez Mendoza

Florida International University

DOI: $10.25148 /$ etd.FI14031609

Follow this and additional works at: https://digitalcommons.fiu.edu/etd

Part of the Civil and Environmental Engineering Commons

\section{Recommended Citation}

Alvarez Mendoza, Patricio, "Evaluating the effectiveness of signal timing optimization based on microscopic simulation evaluation" (2008). FIU Electronic Theses and Dissertations. 1271.

https://digitalcommons.fiu.edu/etd/1271 


\title{
FLORIDA INTERNATIONAL UNIVERSITY
}

Miami, Florida

\section{EVALUATING THE EFFECTIVENESS OF SIGNAL TIMING OPTIMIZATION BASED ON MICROSCOPIC SIMULATION EVALUATION}

\author{
A thesis submitted in partial fulfillment of the \\ requirements for the degree of \\ MASTER OF SCIENCE \\ in \\ CIVIL ENGINEERING \\ by \\ Patricio Alvarez Mendoza \\ 2008
}


This thesis, written by Patricio Alvarez Mendoza, and entitled Evaluating the Effectiveness of Signal Timing Optimization Based on Microscopic Simulation Evaluation, having been approved in respect to style and intellectual content, is referred to you for judgment.

We have read this thesis and recommend that it be approved.

Date of Defense: March 27, 2008

The thesis of Patricio Alvarez Mendoza is approved.

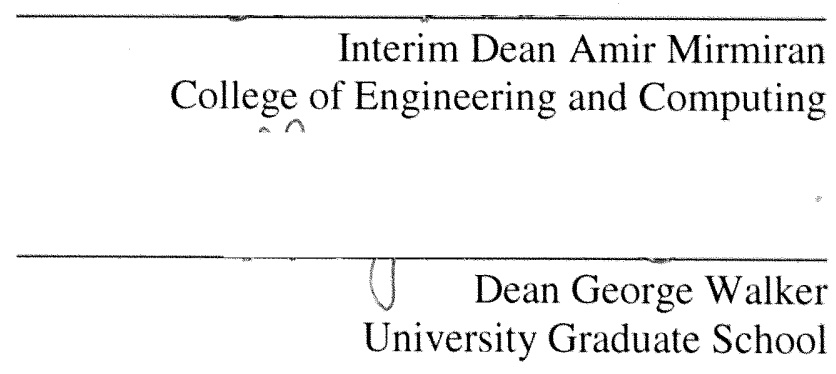

Florida International University, 2008 


\section{DEDICATION}

I dedicate this thesis to Carolina. Without her patience, understanding, support, and most of all love, the completion of this work would not have been possible. 


\section{ACKNOWLEDGMENTS}

I wish to thank to my major professor, Dr. Mohammed Hadi. From the beginning he has had confidence in my intellectual abilities, not only to complete a degree, but also to go further in more challenging pursuits. His wisdom has inspired this thesis and helped me refine the way in which I communicate its results. 


\author{
ABSTRACT OF THE THESIS \\ EVALUATING THE EFFECTIVENESS OF SIGNAL TIMING OPTIMIZATION \\ BASED ON MICROSCOPIC SIMULATION EVALUATION \\ by \\ Patricio Alvarez Mendoza \\ Florida International University, 2008 \\ Miami, Florida \\ Professor Mohammed Hadi, Major Professor
}

The optimization of the timing parameters of traffic signals provides for efficient operation of traffic along a signalized transportation system. Optimization tools with macroscopic simulation models have been used to determine optimal timing plans. These plans have been, in some cases, evaluated and fine tuned using microscopic simulation tools. A number of studies show inconsistencies between optimization tool results based on macroscopic simulation and the results obtained from microscopic simulation. No attempts have been made to determine the reason behind these inconsistencies. This research investigates whether adjusting the parameters of macroscopic simulation models to correspond to the calibrated microscopic simulation model parameters can reduce said inconsistencies. The adjusted parameters include platoon dispersion model parameters, saturation flow rates, and cruise speeds. The results from this work show that adjusting cruise speeds and saturation flow rates can have significant impacts on improving the optimization/macroscopic simulation results as assessed by microscopic simulation models. 


\section{TABLE OF CONTENTS}

CHAPTER

PAGE

I.

INTRODUCTION 1

Problem Description ................................................................. 1

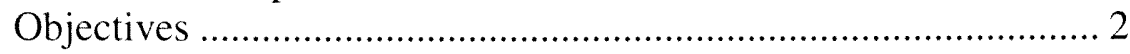

Thesis Organization ................................................................. 3

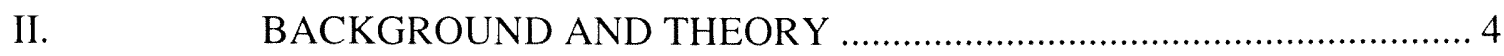

Signal Optimization ….............................................................. 4

Traffic Models .......................................................................... 5

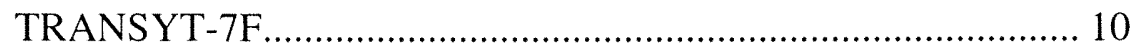

TRANSYT-7F Speed Modeling ......................................... 11

TRANSYT-7F Saturation Flow Rate Modeling .................. 12

TRANSYT-7F Platoon Dispersion Modeling ..................... 14

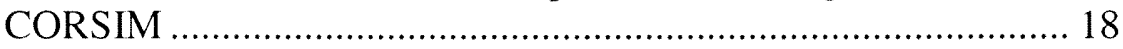

CORSIM Speed Modeling …….................................................. 20

CORSIM Queue Discharge Headway Rate Modeling.................. 21

CORSIM Platoon Dispersion Modeling ...................................... 22

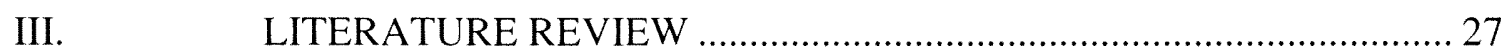

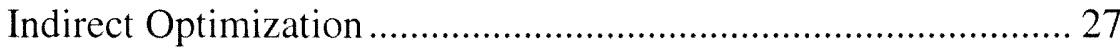

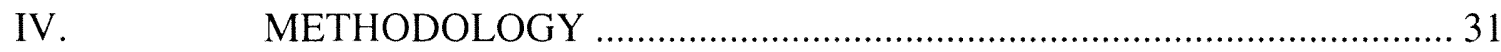

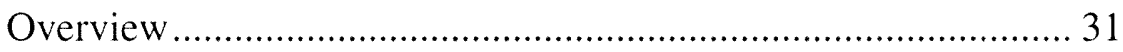

TRANSYT-7F Optimization Parameters.................................... 31

Case Study Description........................................................... 33

Parameter Adjustments ............................................................... 34

Assessment of Plan Performance................................................ 44

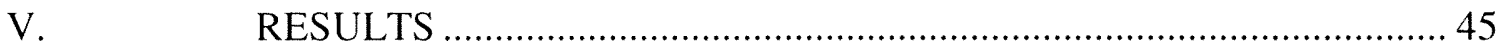

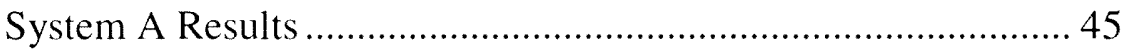

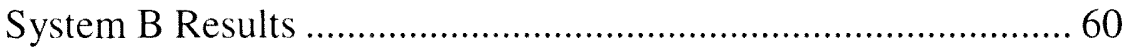

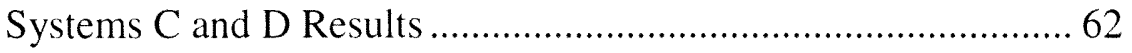

VI. CONCLUSIONS AND RECOMMENDATIONS .................................. 64

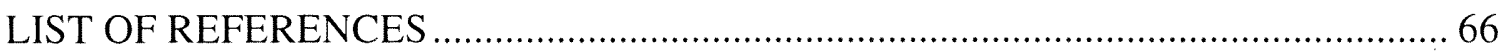




\section{LIST OF TABLES}

TABLE

PAGE

Table 1: Free Flow Multipliers as a Function of Driver Type …...................................... 20

Table 2: Default Distribution of Multipliers for Discharge Headway Percentages.......... 21

Table 3: Default Distribution of Multipliers for Start-up Lost-time Percentages.............. 22

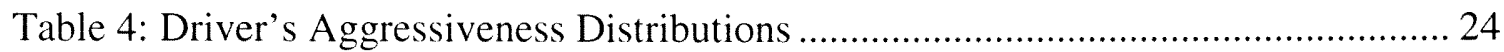

Table 5: Cruise Speed for Different Systems From CORSIM Runs ................................ 38

Table 6: Default and Adjusted Time Headways and Saturation Flow Rates for Different Systems.

Table 7: Robertson's Platoon Dispersion Parameters Different Sources 43 


\section{LIST OF FIGURES}

FIGURE

PAGE

Figure 1: Example of the Use of Arrival Profiles for Delay Computation...... 5

Figure 2: Example of Arrival Patterns Based on Robertson's Platoon Dispersion

Model 6

Figure 3: Example of Arrival Patterns Estimated in CORSIM........................................ 8

Figure 4: Arrival Rate at Stop Bar Based on Robertson's Platoon Dispersion Model, Lenght $=250 \mathrm{ft}$ 15

Figure 5: Arrival Rate at Stop Bar Based on Robertson's Platoon Dispersion Model, Lenght $=500 \mathrm{ft}$ 16

Figure 6: Arrival Rate at Stop Bar Based on Robertson's Platoon Dispersion Model, Lenght $=1000 \mathrm{ft}$ 16

Figure 7: Effect of Alpha in the Predicted Arrival Rate 17

Figure 8: CORSIM Platoon Dispersion Model, Default Parameters Length $=250 \mathrm{ft} \ldots . . .23$

Figure 9: CORSIM Platoon Dispersion Model, Default Parameters Length $=500 \mathrm{ft} \ldots \ldots .23$

Figure 10: CORSIM Platoon Dispersion Model, Default Parameters Length $=1000 \mathrm{ft} . .24$

Figure 11: CORSIM Platoon Dispersion Default Driver Aggressiveness, Link Length $1000 \mathrm{ft}$.....

Figure 12: CORSIM Platoon Dispersion Uniform Driver Aggressiveness, Link Length $1000 \mathrm{ft}$..... 25

Figure 13: CORSIM Platoon Dispersion Anti-Default Driver Aggressiveness, Link

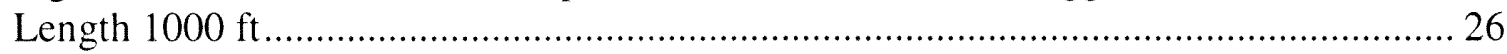

Figure 14: General Procedure for the Collection of the Measures of Performance........... 32

Figure 15: System A Geometry and Operational Set Up............................................... 35

Figure 16: System B Geometry and Operational Set Up ................................................. 35

Figure 17: System C Geometry and Operational Set Up.............................................. 36 
Figure 18: System D Geometry and Operational Set Up

Figure 19: Comparison of TRANSYT-7F and CORSIM Delay Estimates, Link

Length $250 \mathrm{ft}$. 46

Figure 20: Comparison of TRANSYT-7F and CORSIM Delay Estimates, Link Length $500 \mathrm{ft}$. 46

Figure 21: Comparison of TRANSYT-7F and CORSIM Delay Estimates, Link Length $1000 \mathrm{ft}$.

Figure 22: Comparison of TRANSYT-7F and CORSIM Delay Estimates, Link Length $2000 \mathrm{ft}$..... 47

Figure 23: Relative Difference of TRANSYT-7F and CORSIM in Delay

Estimation, Length $250 \mathrm{ft}$ 48

Figure 24: Relative Difference of TRANSYT-7F and CORSIM in Delay

Estimation, Length $500 \mathrm{ft}$.... 48

Figure 25: Relative Difference of TRANSYT-7F and CORSIM in Delay

Estimation, Length $1000 \mathrm{ft}$

Figure 26: Relative Difference of TRANSYT-7F and CORSIM in Delay

Estimation, Length $2000 \mathrm{ft}$

Figure 27: Average Relative Delay Difference as a Function of Link Length................. 50

Figure 28: Average Relative Delay Difference as a Function of V/C. 50

Figure 29: CORSIM Delay Improvement Estimate all Parameters Adjusted in TRANSYT-7F.

Figure 30: TRANSYT-7F (with adjusted parameters) and CORSIM Delay

Estimation versus Offset for Link Length $2000 \mathrm{ft}$.

Figure 31: TRANSYT-7F (with adjusted parameters) and CORSIM Delay

Estimation versus Offset for Link Length $1000 \mathrm{ft}$ 53

Figure 32: TRANSYT-7F (with adjusted parameters) and CORSIM Delay Estimation versus Offset for Link Length $500 \mathrm{ft}$ 54

Figure 33: TRANSYT-7F (with adjusted parameters) and CORSIM Delay Estimation Versus Offset for Link Length $250 \mathrm{ft}$ 
Figure 34: CORSIM and TRANSYT-7F Optimal Offsets 55

Figure 35: CORSIM - TRANSYT-7F Offset Difference as a Function of Link Length 56

Figure 36: TRANSYT-7F and CORSIM Arrival Pattern for Link Length $1000 \mathrm{ft}$ 57

Figure 37: TRANSYT-7F and CORSIM Arrival Pattern for Link Length $2000 \mathrm{ft}$ 57

Figure 38: CORSIM and TRANSYT-7F Optimal Offsets Considering Speed Correction 58

Figure 39: TRANSYT-7F and CORSIM Relative Difference in Optimal Offset Estimation 59

Figure 40: CORSIM Delay Estimate for Different Adjustment Strategies 60

Figure 41: TRANSYT-7F and CORSIM Average Link Delay Estimation 61

Figure 42: CORSIM and TRANSYT-7F Delay Estimation versus Offset in the System 62

Figure 43: CORSIM Delay Estimation Different Adjustment Strategies in TRANSYT-7F. 


\section{INTRODUCTION}

\section{Problem Statement}

The coordination of traffic signals to improve the performance of signalized arterial corridors and grid networks has been standard practice in the traffic engineering industry. This coordination provides for the efficient operation of traffic signals and has been shown to substantially reduce stopped delay and fuel consumption. The coordination of signals requires the selection of signal timing plans that identify a number of signal timing parameters including the number of phases, intervals and phase lengths, cycle lengths, phase sequences, and offsets. Many methods ranging from the use of simple time-space diagrams to the use of complex signal timing optimization programs are commonly used to assist traffic engineers in achieving this coordination.

Signal timing optimization programs have been developed to identify the optimal timing plans that minimize delays, stops, and fuel consumption and/or maximize progression opportunities between signals. TRANSYT-7F (Traffic Network Study Tool Version 7) (1), PASSER-IV (Progression Analysis and Signal System Evaluation Routine Version IV) (2), and SYNCHRO (3) are examples of existing commercially available signal optimization programs.

Signal optimization programs like TRANSYT-7F have used macroscopic simulation models or equations to assess the values of the objective functions during the optimization process. Previous studies have attempted to evaluate the effectiveness of the resulting timing plans using microscopic simulation tools such as CORSIM (Corridor 
Simulator) (4). It has been found that the optimal signal plans obtained using TRANSYT-7F optimization are not optimal according to CORSIM, as is discussed in the literature review section. However, no attempts have been made to investigate the reason for this difference. One of the main reasons could be the difference in the arrival and departure traffic patterns estimated by the microscopic and macroscopic simulation models, which result in different assessments of the optimized signal plans. This could also be related to the default values used for the macroscopic and microscopic model parameters, such as lost time, saturation flow rate, time headway, speed, gap acceptance, and platoon dispersion parameters, that may not be consistent between the macroscopic and microscopic models used.

\section{Objectives}

This thesis investigates issues associated with the assessment of signal timing plans based on microscopic simulation model results. The specific objectives of the study are:

- To compare the arrival and departure patterns and the performance measures estimated by the TRANSYT-7F macroscopic simulation model with those obtained using the CORSIM microscopic simulation model, when using the default adjustment parameters of both models.

- To examine if fine tuning the platoon dispersion, cruise speed, and saturation flow rate parameters in the macroscopic simulation model could result in a better correspondence between the arrival and departure patterns estimated by the macroscopic and microscopic simulation models. 
- To examine the effects of fine tuning the platoon dispersion and saturation flow rate parameters in the macroscopic simulation model on the estimated effectiveness of the optimized plans as assessed by the microscopic simulation program.

- To examine the effects of the degree of saturation in the network and the spacing between signals on the results of the study.

\section{Thesis Organization}

This thesis has been organized into seven chapters. Chapter One provides a general overview of the work done and a set of objectives to be accomplished at the end of this research. Chapter Two introduces theoretical aspects and details regarding traffic signal optimization and assessment and the models used for these purposes. Chapter Three reviews the literature related to this study. Chapter Four describes the methodology and the study cases used for the purposes of this study. Chapter Five presents the results of this study and a discussion about their validity. Chapter Six summarizes the main outcomes of the study and provides final recommendations about the assessment of signal timing plans using microsimulation. 


\section{BACKGROUND AND THEORY}

\section{Signal Optimization}

There are multiple approaches available to solving the traffic signal optimization problem. The objective function definition and optimization algorithm used are key elements for identifying optimal signal timing. Another important element is the use of evaluation/simulation models by the optimization program to assess the alternative solutions developed during optimization based on the selected objective function. The most widely used objective functions are either total delay or a weighted combination of total delay and the number of stops.

By modeling the arrivals and departure patterns, microscopic simulation models can estimate the delay, number of stops, and queue length caused by a specific signal timing plan scheme. Figure 1 shows the arrival profile at a downstream intersection and how it can be used to compute the delay associated with two offset values for the same effective green periods. The arrivals profile at this location is obtained by the use of a platoon dispersion model. In each case, the dashed area corresponds to vehicles not served as they arrive at the signal and that have to wait at the signal as a result.

Optimization programs use macroscopic models to assess delays based on arrival and departure profiles, similar to those shown in Figure 1. 
Indirect methods that combined the optimization/macroscopic simulation process mentioned above with microscopic simulation have also been used. The indirect optimization methods consist of first using macroscopic-simulation/optimization models to determine initial timing plans and later fine tuning the timings based on the results from modeling the network in a microscopic-simulator. This approach combines the computational efficiency of the macroscopic-simulation and the accuracy of the microscopic-simulation approach.

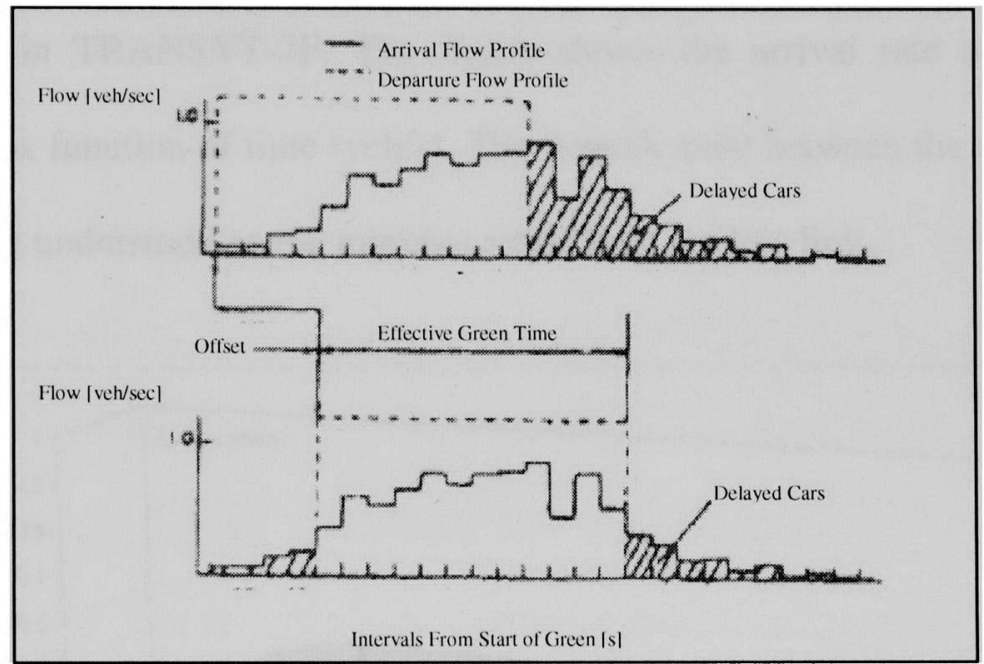

Figure 1: Example of the Use of Arrival Profiles for Delay Computation

\section{Traffic Models}

Macroscopic simulation models predict arrival patterns based on the volume released from an upstream intersection at each time step, link travel time, and a platoon dispersion model. The departure patterns are obtained based on arrival rates, saturation flow rates, and the green time at each time interval. The arrival pattern is a function of how vehicles proceed farther away from the release point at the upstream intersection. The platoons released from upstream intersections are dispersed due to slower than average and faster 
than average vehicles. This phenomenon is known as platoon dispersion. In particular, TRANSYT-7F uses a platoon dispersion algorithm developed by Robertson to model the dispersion of traffic along the link and it is deeply discussed in Reference 5. Depending on the software, other optimization software may or may not consider platoon dispersion in their estimation of arrival patterns.

As an illustrative example, Figure 2 shows a typical arrival pattern based on Robertson's model for a system with a link $250 \mathrm{ft}$ long. The model parameters used were the default values used in TRANSYT-7F. The figure shows the arrival rate at the downstream intersection as function of time $(\mathrm{veh} / \mathrm{s})$. The time ( $\mathrm{x}$ axis) between the origin and the first arrival can be understood as the minimal travel time for this link.

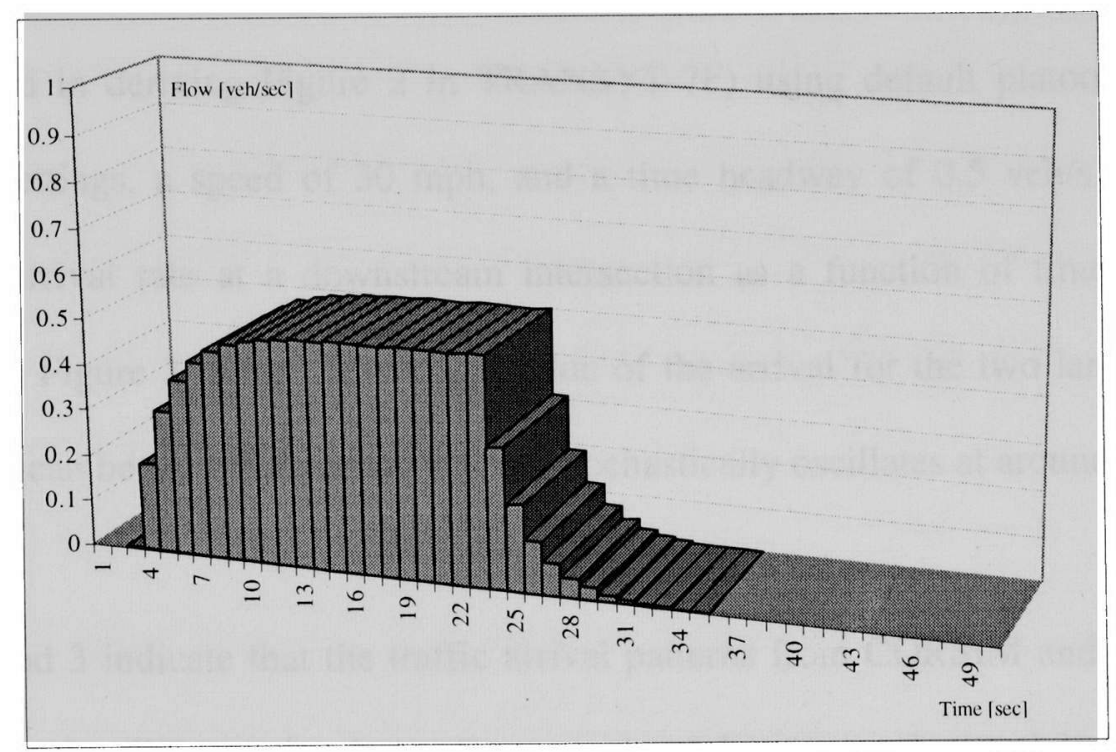

Figure 2: Example of Arrival Patterns Based on Robertson's Platoon Dispersion Model

In microscopic simulation models like in CORSIM, a series of stochastic processes at the microscopic level are used to simulate traffic flow. These models require detailed signal 
timing, volume, and geometric information, as well as a significant calibration effort. The models track each simulated vehicle each second (or fraction of a second) based on carfollowing, lane changing, gap acceptance, and other microscopic simulation logics, as well as a driver's probable responses to the physical traffic environment. In CORSIM, platoon dispersion is inherent in the modeling of traffic flows because the vehicles affect each other due to car-following logic. This simulates real world situations by maintaining safe stopping distances between vehicles, taking into account driver aggressiveness and vehicle performance. The arrival and departure patterns are determined as a result of the microscopic simulation process.

As a example, a graphical representation of a typical CORSIM platoon dispersion is shown in Figure 3 for a system composed of one 250-ft-long link (with similar settings to the one used in deriving Figure 2 in TRANSYT-7F) using default platoon dispersion parameter settings, a speed of $30 \mathrm{mph}$, and a time headway of $0.5 \mathrm{veh} / \mathrm{s}$. This figure shows the arrival rate at a downstream intersection as a function of time. The result presented in Figure 3 is for the average value of the arrival for the two lanes along the approach. It can be seen that the arrival rate stochastically oscillates at around $0.5 \mathrm{veh} / \mathrm{s}$.

Figures 2 and 3 indicate that the traffic arrival patterns from CORSIM and TRANSYT$7 \mathrm{~F}$ appear to be different in shape. Because the delays are calculated based on these patterns, this difference is expected to result in a difference in the estimation of delay between the two models. This, in turn, may result in solutions that appear to be optimal 
according to TRANSYT-7F optimization, but that are assessed as suboptimal according to CORSIM.

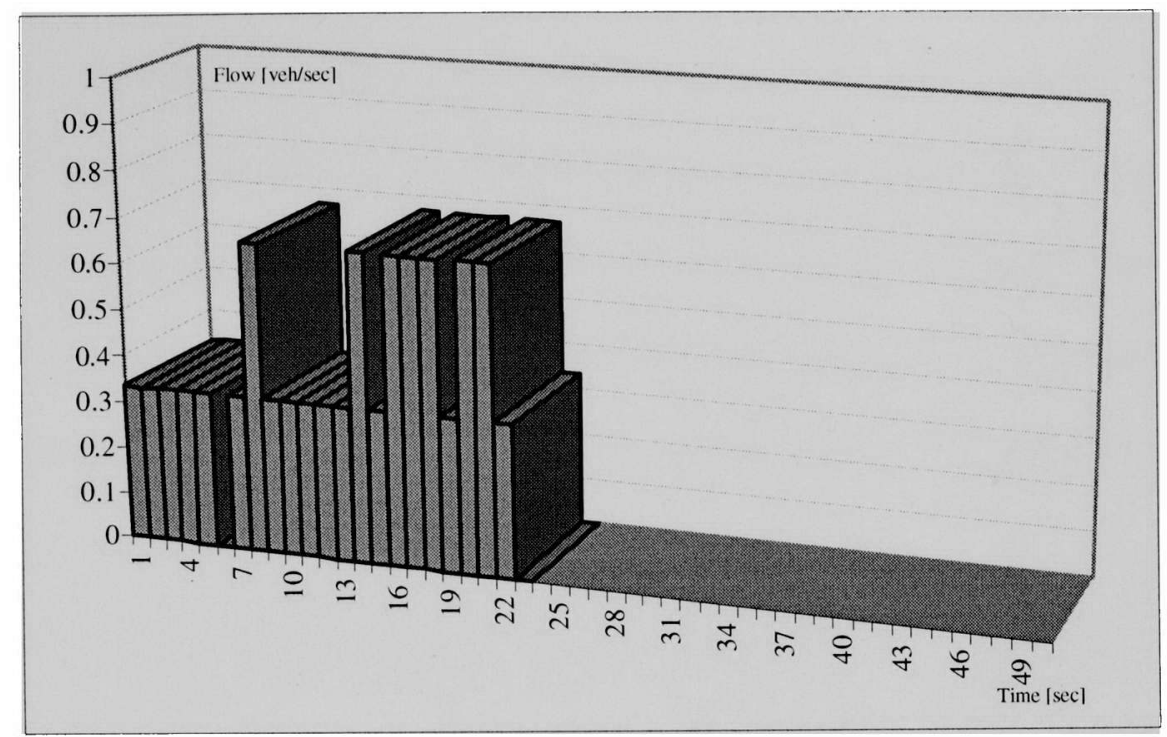

Figure 3: Example of Arrival Patterns Estimated in CORSIM

An additional reason for inconsistency in the assessment of the optimal solutions between the two types of models could be the treatment of the headway (saturation flow), which is deterministic in TRANSYT-7F and stochastic in CORSIM. A third reason could be the difference in the assessment of the cruise speed between TRANSYT-7F and CORSIM, as described later in this document.

As mentioned before, there are several software programs that can be utilized to optimize and simulate signal timing plans for arterial streets. This study will utilize TRANSYT-7F as the optimization/macroscopic simulation model and CORSIM as the microscopic simulation model. TRANSYT-7F and CORSIM are widely used in the United States, and 
there is a large amount of research and practice based on which to validate the quality of their results. 


\section{TRANSYT-7F}

TRANSYT-7F (1) is a traffic simulation and signal timing optimization program that is primarily applied to signal timing design and optimization. TRANSYT-7F features Hill Climbing and Genetic Algorithm optimization algorithms of signal timing. In addition, while other models are limited to analyzing four or five intersection approaches, an advantage of TRANSYT-7F is that there is no practical limitation to the number of approaches that can be simulated. Since version 8 of the program was first developed, the program options have allowed TRANSYT-7F to simulate and optimize oversaturated conditions.

The TRANSYT-7F optimization process features the availability of multiple optimization objective functions (e.g., involving combinations of progression opportunities, delay, number of stops, fuel consumption, throughput, and queuing); an extensive ability to customize the optimization process; and the ability to optimize cycle length, phasing green splits, and offsets.

The original TRANSYT model was developed by the Transport Research Laboratory (formerly Transport and Road Research Laboratory) in the United Kingdom. TRANSYT, version 7 was "Americanized" for the Federal Highway Administration (FHWA), hence the "7F." The TRANSYT-7F program and the original TRANSYT-7F manual were developed for the Federal Highway Administration (FHWA) under the National Signal Timing Optimization (NSTOP) Project by the University of Florida Transportation Research Center (TRC). The TRANSYT model was originally developed by Dr. Dennis 
I. Robertson of the United Kingdom in 1967. The first TRANSYT report was also issued as a TRRL Report, LR 253, which is one of the primary TRANSYT references.

This first version was written in machine code for a Myriad computer. Dr. Robertson and his colleagues converted the program to FORTRAN and continued to make improvements over the years.

Below is a discussion of specific components of TRANSYT-7F modeling that are of particular interest to this study.

\section{TRANSYT-7F Speed Modeling}

According to the TRANSYT-7F User Manual, the link speed is a deterministic parameter that represents the actual average cruise speeds on links. This cruise speed primarily affects the arrival pattern and platoon dispersion, but fuel consumption and travel time outputs are also affected by this parameter. Note that the proper speed entries to be coded should be the actual average cruise speeds. The TRANSYT-7F user manual mentioned that, for optimization runs, the speed entries should be the desired speeds. The effects of delay due to the signal should not be included when estimating the input values. This description of the speed by the user manual is somewhat confusing because cruise speed and desired speed (free flow speed) represent different concepts in traffic engineering. Actually, TRANSYT-7F does not have an internal model to adjust the coded speed according to the actual level of traffic in the system, and it is well known that as the traffic increases the cruise speed decreases. Independently of what is stated in the User's 
Manual, in this work the speed will be considered the actual cruise speed given a specific set of traffic conditions.

\section{TRANSYT-7F Saturation Flow Rate Modeling}

To aid in understanding the Saturation Flow Rate concept, consider a single lane of traffic (or link) with a standing queue at an intersection waiting for the green display. Following the phase change to green for this link, there is a slight delay before the driver of the first vehicle reacts and crosses the stopline. This is the start-up lost time. After several vehicles have crossed the stopline (usually about three vehicles), the queue begins to discharge at essentially a constant rate. This constant rate is called the saturation flow rate. The unit of saturation flow rate is either vehicles per lane per hour of green (vplphg), or the total vehicles per hour of green (vphg) across all lanes. The unit is expressed in "hours of green" because the saturation flow rate represents the number of vehicles that theoretically would be served if a full hour of green time was available. A saturation flow rate of 1895 vplphg would indicate an average 1.9-second front-bumper-to-front-bumper headway between vehicles throughout the hour. Generally speaking, the capacity of a link is much lower than its saturation flow rate because a given link does not receive a green light throughout the period of analysis.

As mentioned before, the saturation flow rate is used by macroscopic traffic flow models to estimate the departure of queues from a link. Along with the volume, travel time between nodes, number of lanes, and timing plan, saturation flow rate is one of the most important factors in determining traffic network performance. Because of this, it is 
important that reasonable estimates of the saturation flow rate be provided by the user. In general, the TRANSYT-7F User's Manual recommends following the techniques for field measurement of saturation flow rates as discussed in the Highway Capacity Manual (HCM) 2000 (6). However, in practice, a large proportion of signal optimization users estimate rather than measure the saturation flow rates for the purpose of signal timing optimization.

The HCM recommends a "base" (unadjusted) saturation flow rate of 1900 (vplphg). In estimating saturation flow rates, adjustments to the base saturation flow rates need to be made to account for prevailing conditions. The HCM also includes recommended values for these adjustments. The HCM procedures for estimating saturation flow rates are generally used by signal timing optimization users, although to different levels of detail depending on the user(s).

Note that the HCM 2000 presents adjustment factors for the saturation flow rates of left and right turn movements. The reduction in saturation flow rates for protected turns accounts for the more sluggish departures of vehicles from turning queues. Presumably, it is more difficult for turning vehicles to stay in their lane, and, thus, larger headways are required. Assuming right-hand-side driving, drivers making right turns are typically assumed to travel at a speed of approximately $9 \mathrm{mph}(14.4 \mathrm{kmph})$, or $85 \%$ of the ideal saturation flow rate. Left-turners are typically assumed to travel at a speed of approximately $15 \mathrm{mph}(24.0 \mathrm{kmph})$, or $95 \%$ of the ideal saturation flow rate. 
As mentioned earlier in this thesis, TRANSYT-7F uses a platoon dispersion algorithm developed by Robertson to model the dispersion of traffic along a link. Because of the simplicity of applying a recursive formulation, Robertson's model has become the standard platoon dispersion model and has been incorporated into a number of software packages other than TRANSYT-7F.

The basic Robertson's recursive platoon dispersion model takes the following mathematical form:

$q_{t}^{\prime}=F q_{t-T}+(1-F) q_{t-\Delta t}^{\prime}$

$F=\frac{1}{1+\alpha \beta T_{a}}$

where

$q_{t} \quad$ : Flow rate over a time step $\Delta \mathrm{t}$ arriving at the downstream signal at time $\mathrm{t}$ (vehicles per time step unit),

$q_{t-T} \quad$ : Discharging flow over a time step $\Delta \mathrm{t}$ observed at the upstream signal at time $\mathrm{t}-\mathrm{T}$ (vehicles per time step unit),

$q_{t-\Delta t}^{\prime} \quad$ : Flow rate over a time step $\Delta \mathrm{t}$ arriving at the downstream signal at time $\mathrm{t}-\Delta \mathrm{t}$ (vehicles per time step unit), 
$\Delta t \quad$ : Modeling time step duration (units of time steps),

F : Smoothing factor (units of time $\operatorname{steps}^{-1}$ ),

$\alpha \quad$ : Platoon dispersion factor (unitless),

$\beta \quad$ : Travel time factor (unitless), and

$T_{a} \quad$ : Mean roadway travel time (units of time steps),

Figures 4 through 6 show typical arrival patterns calculated based on Roberson's platoon dispersion model using the TRANSYT-7F default parameters [alpha $=0.35$ and beta $=0.8$ in Equations (2.1) and (2.2)]. The arrival rates at 250-ft (Figure 4), 500-ft (Figure 5), and 1000 -ft (Figure 6) downstream flows are predicted based on a $0.5-\mathrm{veh} / \mathrm{s}$ release rate upstream. The profiles show triangular shapes, with the platoon becoming less compressed as it travels further downstream of the upstream intersection.

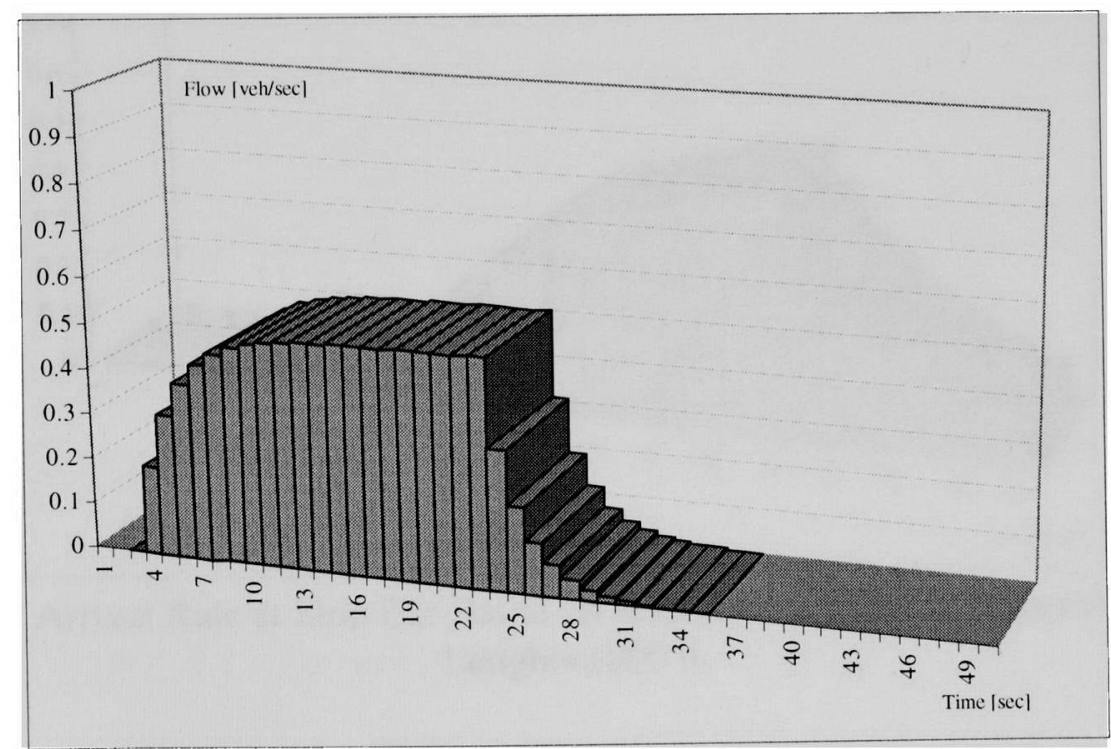

Figure 4: Arrival Rate at Stop Bar Based on Robertson’s Platoon Dispersion Model, Lenght $=250 \mathrm{ft}$ 


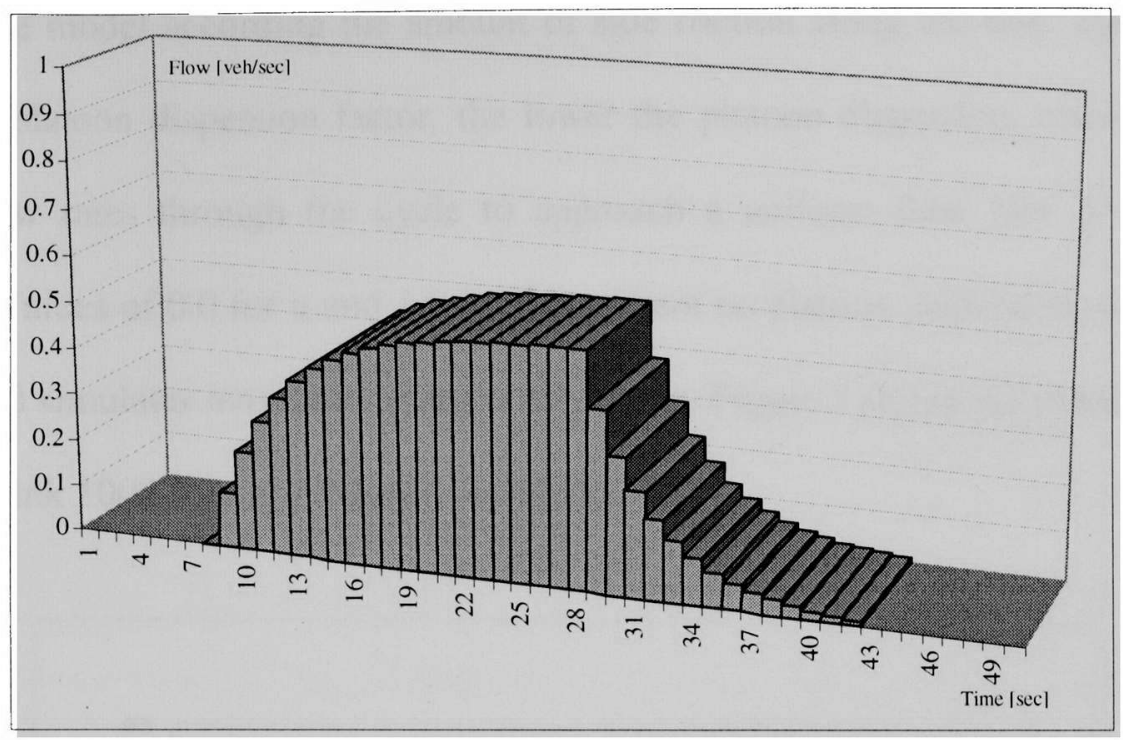

Figure 5: Arrival Rate at Stop Bar Based on Robertson's Platoon Dispersion Model, Lenght $=500 \mathrm{ft}$

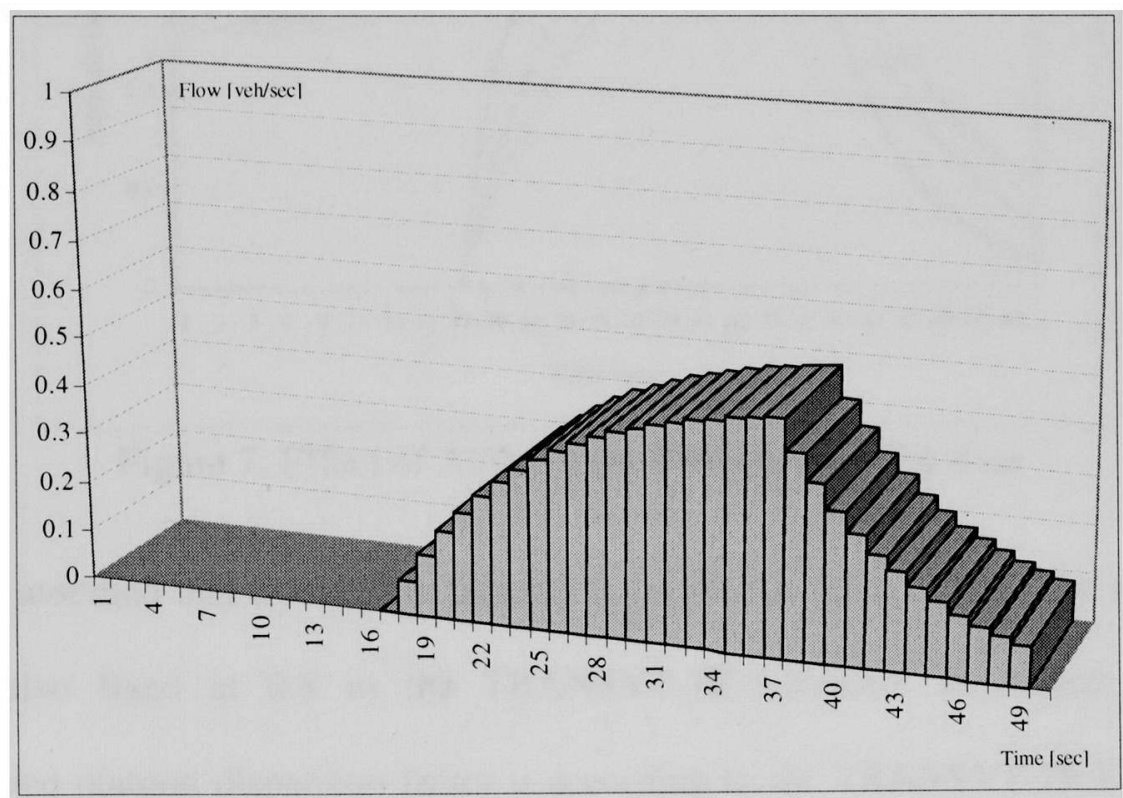

Figure 6: Arrival Rate at Stop Bar Based on Robertson's Platoon Dispersion Model, Lenght $=1000 \mathrm{ft}$

As mentioned, TRANSYT-7F software defaults for the platoon dispersion parameters $\alpha$ and $\beta$ are 0.35 and 0.8 , respectively. These values are suggested in the program user guide for urbanized arterials in the United States. The platoon dispersion factor $\alpha$ is used 
to adjust the model according the amount of side friction along the link. The lower the value of a platoon dispersion factor, the lower the platoon dispersion, causing the step interval flow rates through the cycle to approach a uniform flow rate as travel time increases. Values of 0.0 for $\alpha$ and 1.0 for $\beta$ represent no platoon dispersion, while 1.0 for both $\alpha$ and $\beta$ simulates maximum platoon dispersion. Figure 7 shows the effect of varying alpha on a link $1000 \mathrm{ft}$ long produced in our study.

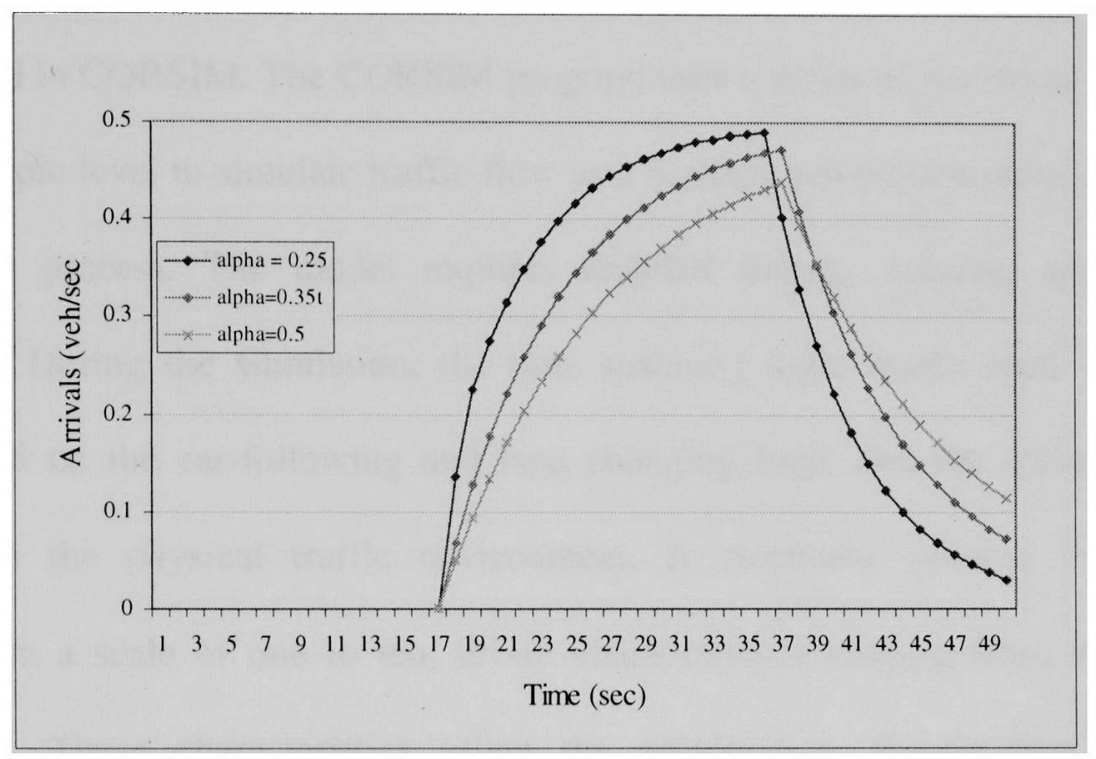

Figure 7: Effect of Alpha in the Predicted Arrival Rate

Robertson assumed that travel time factor $\beta$ is fixed at a value of 0.8 . The value of this factor is also fixed at 0.8 in the TRANSYT-7F software. As stated above, the recommended platoon dispersion factor $\alpha$ according to the TRANSYT-7F User's Guide is between 0.25 and 0.5 , depending on the level of friction along the roadway. As with most models, the application of Robertson's model relies on the appropriate adjustment of its parameters. All versions of the TRANSYT software allow only for the adjustment 
of the platoon dispersion factor $\alpha$ and do not allow for the adjustment of the travel time factor.

\section{CORSIM}

CORSIM is a microscopic time-scanning traffic simulation tool for urban surface streets and freeway networks. Microscopic implies that the model represents detailed movements of each vehicle in the traffic stream for each designated time interval, which is one second in CORSIM. The CORSIM program uses a series of stochastic processes at the microscopic level to simulate traffic flow as a realistic representation of a real world, time-varying process. The model requires detailed timing, volume, and geometric information. During the simulation, the time scanning logic tracks each vehicle each second based on the car-following and lane changing logic and the driver's probable responses to the physical traffic environment. A stochastic process is applied to determine, on a scale of one to ten, driver characteristics ranging from aggressive to conservative. These characteristics affect the acceleration, deceleration, and speed attributes of vehicles in the network. The model assigns different speed, acceleration, and deceleration and headway attributes to each vehicle as it enters the network based on the driver's characteristics assigned to that vehicle.

CORSIM includes stochastic models, which means that driver and vehicle characteristics are assigned randomly. The Measures of Effectiveness (MOEs) obtained from a simulation are different for different coded seed numbers. For example, one set of random number seeds may result in three very conservative drivers driving side by side 
on a three-lane roadway blocking more aggressive drivers behind them. In this case, the resulting MOE would reflect a lower average speed than the expected average values. Relying on the MOEs generated from a single run of CORSIM may be misleading. To gain a better understanding of a network, the performance of the network should be simulated several times using different sets of random number seeds. The resulting distribution of MOEs should then be an accurate representation of network performance.

In CORSIM, each vehicle is identified as belonging to a fleet (auto, carpool, truck, or bus) and to a type. Up to nine different types of vehicles (with different operating and performance characteristics) can be specified, thus defining the four vehicle fleets. Furthermore, a "driver behavioral characteristic" (passive or aggressive) is assigned to each vehicle. The kinematics properties (speed and acceleration), as well as a vehicle's status (queued or moving), are determined by the simulation models. Turning movements are assigned stochastically, as are free-flow speeds, queue discharge headways, and other behavioral attributes. As a result, each vehicle's behavior can be simulated in a manner reflecting real world processes.

Each time a vehicle moves, its position (both lateral and longitudinal) on the link and its relationship to other vehicles nearby are recalculated, as are its speed, acceleration, and status.

CORSIM accumulates data every time step (every second). These accumulated data are used at the end of each time period (specified by the analysis) to produce the reported MOEs. 
The cruise speeds, saturation flow rates, and platoon dispersions are products of the traffic flow modeling in CORSIM. This is because vehicles affect each other due to carfollowing logic, simulating real world situations by maintaining safe stopping distances between adjacent vehicles.

\section{CORSIM Speed Modeling}

In CORSIM, the speed is modeled as a distribution of free-flow speed percentages. The values of these percentages range from $75 \%$ to $127 \%$ of the mean free-flow speed. These percentages are used to calculate the free-flow speeds of individual vehicles. CORSIM is able to adjust these values to reflect the interaction between vehicles. The distribution itself is a description of the driver population, and, after it is calculated, CORSIM can also take in account different population distributions as well as different values of the free-flow speed. Table 1 shows the free-flow speed distribution as a function of driver type.

Table 1: Free Flow Multipliers as a Function of Driver Type

\begin{tabular}{|l|c|c|c|c|c|c|c|c|c|c|}
\hline Driver Type & 1 & 2 & 3 & 4 & 5 & 6 & 7 & 8 & 9 & 10 \\
\hline $\begin{array}{l}\text { Percentage Multiplier of Free- } \\
\text { Flow Speed }\end{array}$ & 75 & 81 & 91 & 94 & 97 & 100 & 107 & 111 & 117 & 117 \\
\hline
\end{tabular}

In the Driver Type distribution table, the sum of all of the entries must equal 1000. Fatal program errors occur if any of the entries are negative, or if the sum of all of the entries does not equal 1000. It is possible to adjust the default driver distribution to replicate the local driver distribution. 
As with speed, CORSIM models the queue discharge headway rates as distributions that depend both on the driver characteristic distribution mentioned above and the specific link being modeled. Up to four queue discharge headway distributions can be defined, each associated with a link type.

As each queued vehicle moves up to the stop line, it is assigned a delay until discharged (in tenths of a second), reflecting its queue discharge headway. This headway is obtained by multiplying the mean queue headway specified for the link by a percentage. This percentage is extracted from a decile distribution that applies to that "type" of link. The vehicle's driver characteristic is used as an index for referencing the proper element in the distributions.

As example, Table 2 shows the embedded default values for two default distributions of discharge headways.

Table 2: Default Distribution of Multipliers for Discharge Headway Percentages

\begin{tabular}{|l|c|c|c|c|c|c|c|c|c|c|}
\hline Driver Type & 1 & 2 & 3 & 4 & 5 & 6 & 7 & 8 & 9 & 10 \\
\hline Distribution Code 1 & 170 & 120 & 120 & 110 & 100 & 100 & 90 & 70 & 70 & 50 \\
\hline Distribution Code 2 & 180 & 140 & 120 & 110 & 100 & 90 & 80 & 70 & 60 & 50 \\
\hline
\end{tabular}

There are no internal default values specified for distribution codes 3 and 4 . These can be added as needed.

The first vehicle in a queue when the signal turns to green experiences (start-up) lost time. Lost time (in tenths of a second) is computed by referencing a decile distribution defined by the distribution code. The vehicle's driver characteristic is used as an index for 
referencing the proper element in the distribution. The start-up lost-time percentages array contains four such distributions, one for each of four distribution codes. Elements of the array contain percentage values applied to the specified mean lost time. Table 3 shows the embedded default values for two default distributions of start up lost time.

Table 3: Default Distribution of Multipliers for Start-up Lost-time Percentages

\begin{tabular}{|l|l|l|l|l|l|l|l|l|l|l|}
\hline Driver Type & 1 & 2 & 3 & 4 & 5 & 6 & 7 & 8 & 9 & 10 \\
\hline Distribution Code 1 & 218 & 140 & 125 & 118 & 102 & 86 & 78 & 63 & 47 & 23 \\
\hline Distribution Code 2 & 258 & 190 & 143 & 114 & 95 & 76 & 57 & 38 & 29 & 0 \\
\hline
\end{tabular}

\section{CORSIM Platoon Dispersion Modeling}

As mentioned earlier, the CORSIM program uses a series of stochastic processes at a microscopic level to simulate traffic flow as a realistic representation of a real world, time-varying process. The platoon dispersion is the result of the interaction of more elementary models like the car following model, the lane change model, and the driver population distribution. A graphical representation of typical CORSIM platoon modeling that was derived in this study for illustrative purposes is shown in Figures 8 through 10 . The arrivals rates at $250 \mathrm{ft}$ (Figure 8), $500 \mathrm{ft}$ (Figure 9), and $1000 \mathrm{ft}$ (Figure 10) downstream are predicted based on a $0.5-\mathrm{veh} / \mathrm{s}$ upstream release rate. It can be seen that the farther away the downstream signal, the more spread out the platoon will be. Unlike TRANSYT-7F, the CORSIM model shows a random variation in the flow profile at each second. This is due to the microscopic simulation, which produces multiple platoons throughout the cycle. Also, the formation of mini platoons can be observed at 500 and $1000 \mathrm{ft}$. Another factor is that, in CORSIM, lane changing options allow vehicles to pass each other. 


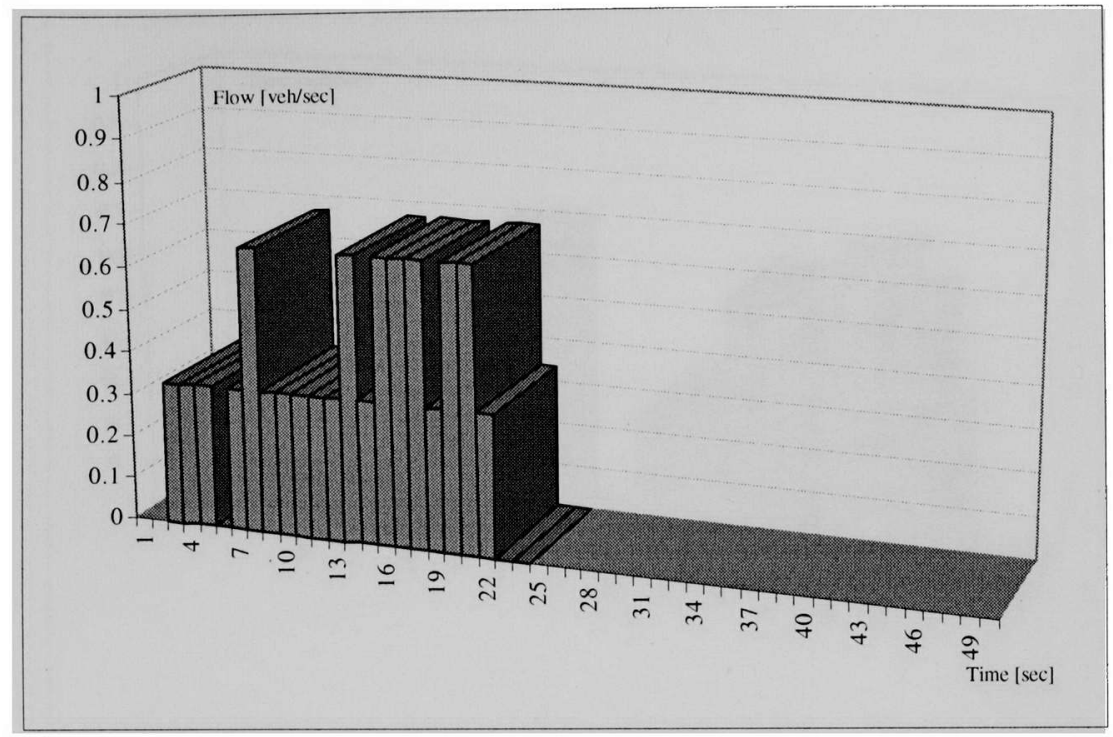

Figure 8: CORSIM Platoon Dispersion Model, Default Parameters Length $=250 \mathrm{ft}$

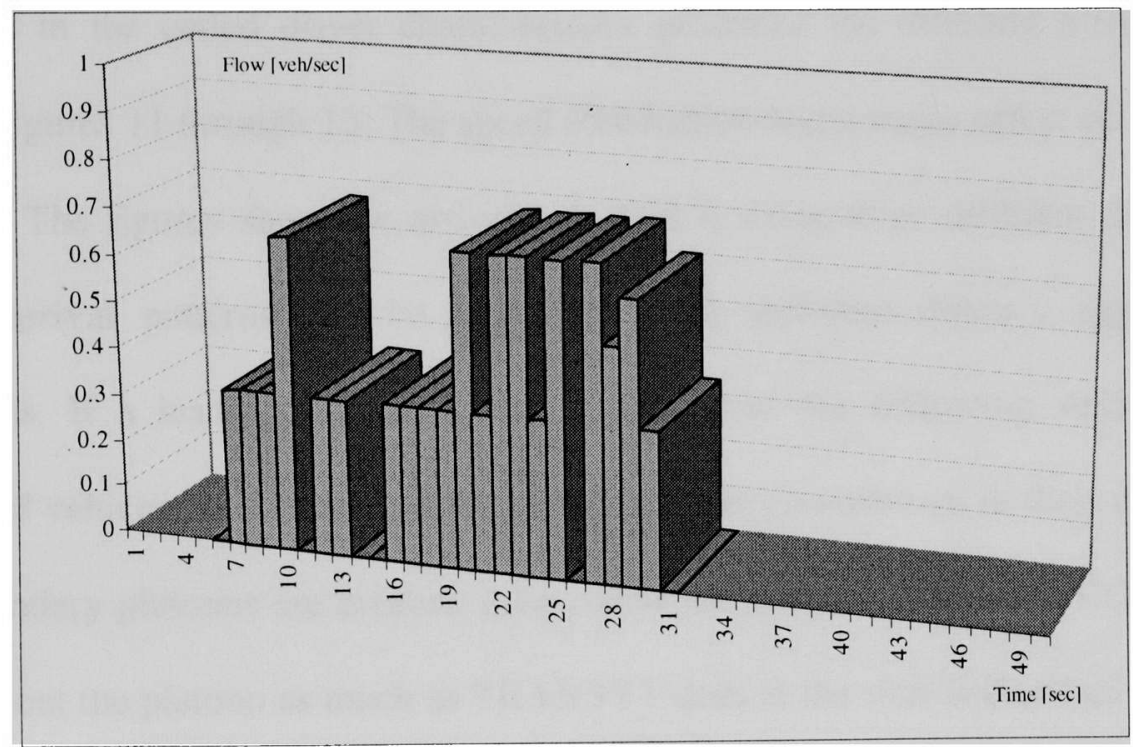

Figure 9: CORSIM Platoon Dispersion Model, Default Parameters Length $=500 \mathrm{ft}$ 


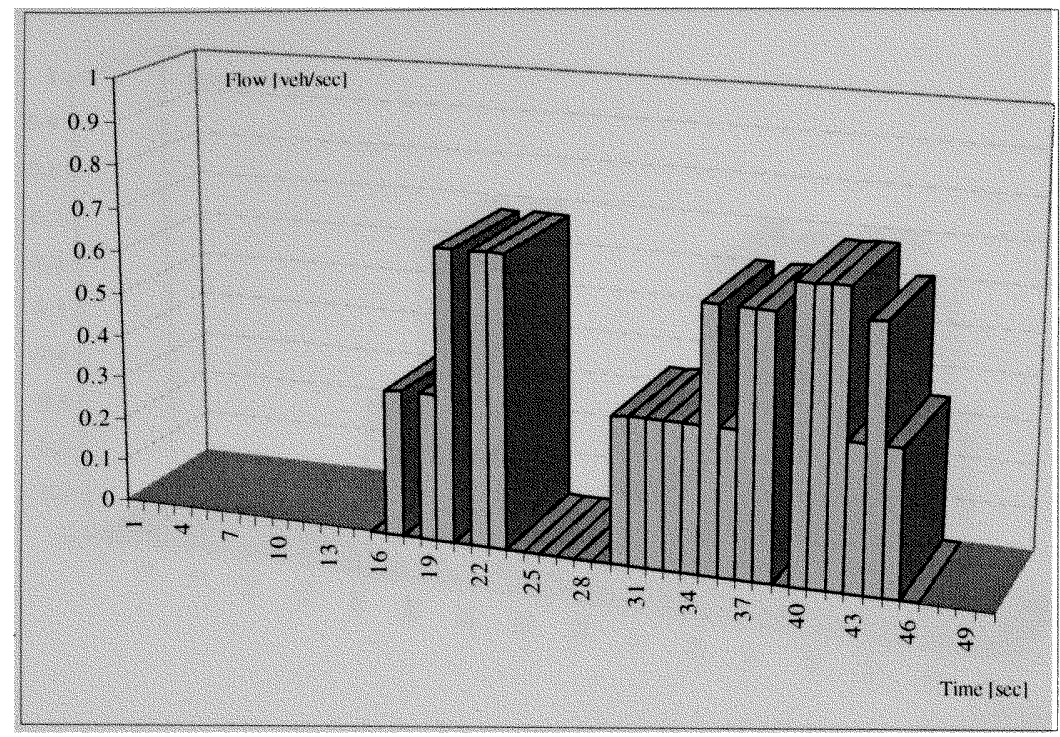

Figure 10: CORSIM Platoon Dispersion Model, Default Parameters Length $=1000 \mathrm{ft}$

Differences in the coded driver characteristics produced the different arrival patterns shown in Figures 11 through 13. The speed distribution had a major effect on the platoon dispersion. The figures show the arrivals at $1000 \mathrm{ft}$ using three different distributions. Different arrival patterns can be associated with different driver's aggressiveness distributions. If a leading vehicle travels slower than the following vehicles then a bunching of vehicles will occur. As the vehicles travel downstream in their concentrated form, secondary platoons are created. Also, when the two are compared, CORSIM does not spread out the platoon as much as TRANSYT does at the start and end of the platoon. The distributions for different driver aggressiveness levels are shown in Table 4.

Table 4: Driver's Aggressiveness Distributions

\begin{tabular}{|l|c|c|c|c|c|c|c|c|c|c|}
\hline Driver Type & 1 & 2 & 3 & 4 & 5 & 6 & 7 & 8 & 9 & 10 \\
\hline Default & 125 & 121 & 116 & 108 & 100 & 97 & 93 & 85 & 80 & 75 \\
\hline Uniform & 100 & 100 & 100 & 100 & 100 & 100 & 100 & 100 & 100 & 100 \\
\hline Reverse Default & 75 & 80 & 85 & 93 & 97 & 100 & 108 & 116 & 121 & 125 \\
\hline
\end{tabular}




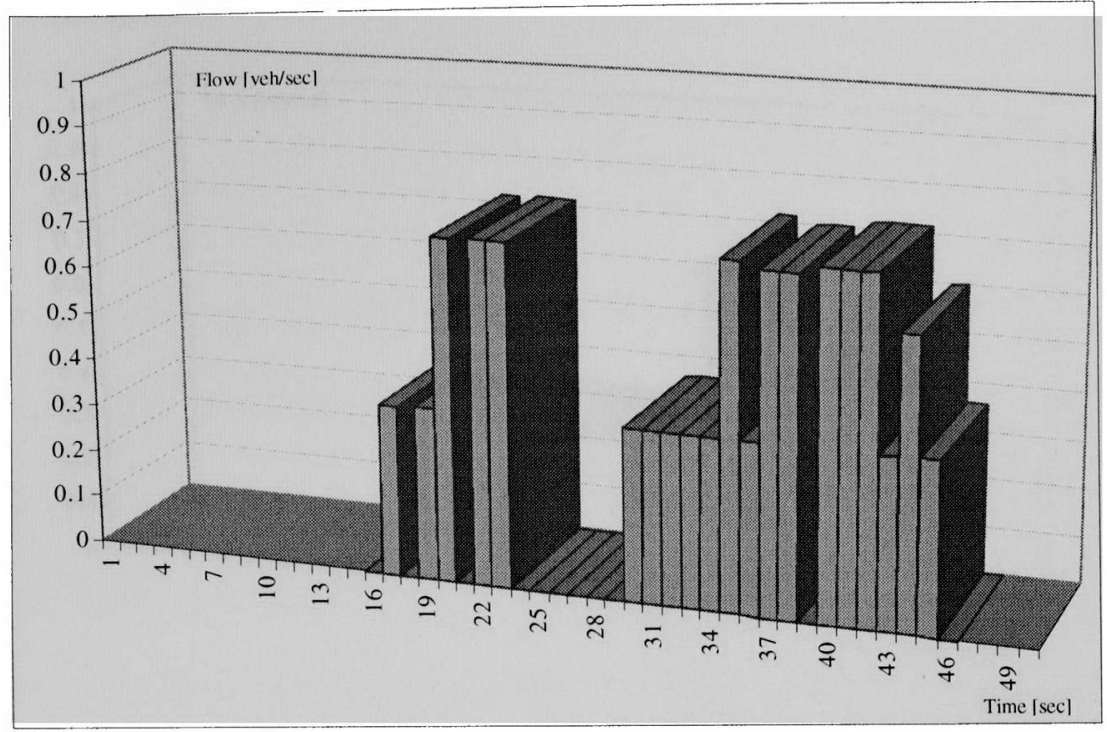

Figure 11: CORSIM Platoon Dispersion Default Driver Aggressiveness, Link Length $1000 \mathrm{ft}$

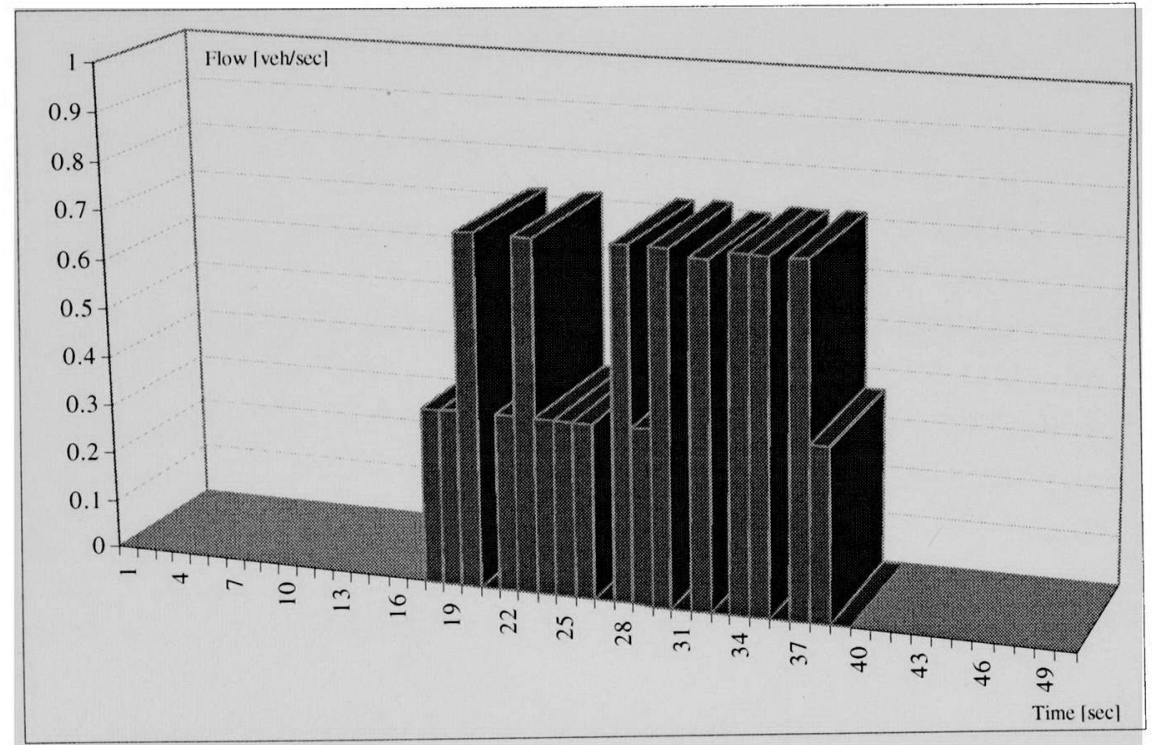

Figure 12: CORSIM Platoon Dispersion Uniform Driver Aggressiveness, Link Length $1000 \mathrm{ft}$ 


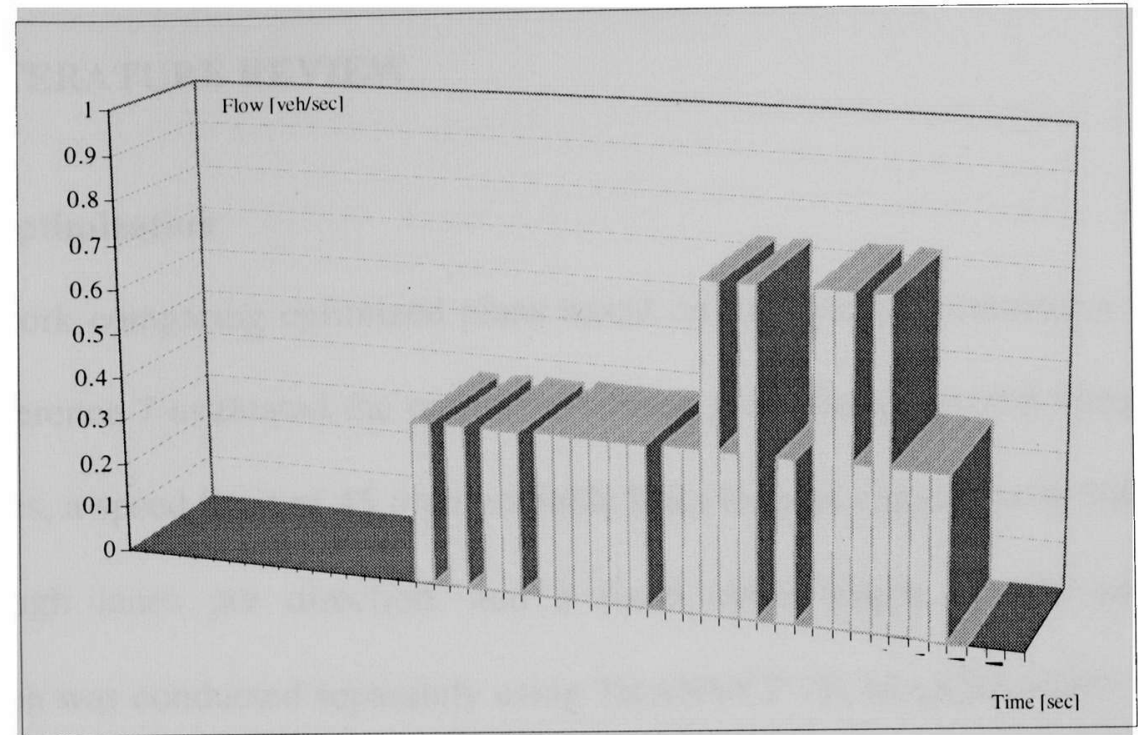

Figure 13: CORSIM Platoon Dispersion Anti-Default Driver Aggressiveness, Link Length $1000 \mathrm{ft}$ 


\section{LITERATURE REVIEW}

\section{Indirect Optimization}

The first work comparing optimized plans based on microscopic simulation dates to the 1980s. Reference 7 evaluated the optimized timing plans for an arterial street with eight intersections, a speed limit of $45 \mathrm{mph}$, variable links lengths ranging from 500 to $3000 \mathrm{ft}$, three through lanes per direction, and a fixed cycle length of 100 seconds. The optimization was conducted separately using TRANSYT-7F, MAXBAND93, PASSERII, and SYNCHRO 2.0. The optimized outputs in this case were the splits and the offsets at each intersection. These outputs were coded in the micro-simulator TRAF-NETSIM, and the different measures of performance for each plan were compared. The comparison results yield the conclusion that there were significant differences in the measures of performance of the micro-simulators, depending on what optimization program was used. In particular, it was observed that MAXBAND performed the best and that TRANSYT7F performed the worst in terms of simulated delays.

Several years after the study mentioned above, another study (8) investigated the extent to which TRANSYT-7F optimized signal plans appeared to be close to optimal when evaluated in a microscopic simulation program. The case study network consisted of nine signalized intersections. CORSIM was validated based on the observed maximum queue lengths per cycle at key intersections. The simulated observed maximum queues frequency distribution over 100 simulations was compared with the observed maximum queue lengths in the field. The optimal plans in TRANSYT-7F were obtained based on 
measures of performance that are directly comparable with those provided by CORSIM, namely, delay, fuel consumption, percent stops, queue time, and throughput. Twelve optimization strategies (objective functions) in TRANSYT-7F were tested to produce the optimized plans. The plans were later simulated in CORSIM 100 times each. For the uncongested condition, correlation coefficients of approximately $0.90,0.71$, and 0.61 were calculated between the results of TRANSYT-7F and CORSIM for delay, fuel consumption, and stops, respectively. Although high degrees of correlation were observed, the actual values of the measures were quite different. The correlation coefficients under congested conditions were substantially smaller than in the uncongested case ( 0.72 for delay, 0.31 for fuel consumption, and 0.53 for stops). Also note that, when implemented in the micro-simulator, the order of the ranking of TRANSYT optimal plans (with different optimization objective functions) changed.

Reference 9 used CORSIM simulation results to compare the performance of the optimal plans from TRANSYT-7F with those obtained from CORSIM using a Genetic Algorithm (GA). In this case, a number of TRANSYT-7F parameters were calibrated, including the link saturation flow rate, the left turn sneakers, and the link free-flow speed. CORSIM was validated by comparing the observed and simulated maximum queue lengths over 100 repetitions. The studied network consisted of nine signalized intersections with most intersections operating under two-phase signal control with permitted left turns.

It was shown that the GA settings consistently outperform TRANSYT-7F strategies, even though the best TRANSYT-7F was selected on the basis of how it performed in 
CORSIM, not in TRANSYT-7F. The CORSIM-based GA optimization not only improved the mean value of the performance measure, but also reduced its variance. The GA settings reduced the simulated delays by about $37 \%$ and the simulated queue time by about $53 \%$.

Reference 10 examined the performance of aging signal plans using an uncongested hypothetical network. The network was composed of nine nodes and variable link lengths between 800 and $1000 \mathrm{ft}$ long, with a six-lane (two-way) arterial street. All of the approaches have 250 -ft-long left turn pockets and 150 -ft-long right turn pockets. The base flows at the entry points were set up to $800 \mathrm{vph}$, with a free-flow speed of $45 \mathrm{mph}$. All of the turning proportions in the network were set to be the same: $78 \%$ through traffic and $11 \%$ left and right turns. The optimal plans were obtained by using optimization/macro-simulation programs and later evaluated in a micro-simulator. In this study case, the selected optimization programs were TRANSYT-7F and SYNCHRO, and the micro-simulators were CORSIM, SIMTRAFFIC, and VISSIM. A direct optimization in microscopic simulation was attempted using the GA in CORSIM. All programs were utilized with the default settings of their parameters. The optimal plans from the optimization/macro-simulator programs were obtained by minimizing a performance index (PI), which is a composite measure of the delay time and stops, two of the most widely used measures of effectiveness for undersaturated traffic conditions. Later, these optimal plans were simulated in the micro-simulators at least ten times each to improve confidence in the results. The results indicate systematically inconsistent outcomes for 
most of the macro-micro optimization combinations. Additionally, the direct optimization in CORSIM did not generate significantly better timing plans than the existing plans. 


\section{METHODOLOGY}

\section{Overview}

This study first examines if fine tuning the platoon dispersion, speed, and saturation flow parameters in the macroscopic simulation model result in better correspondence of the arrival and departure patterns estimated by the macroscopic and microscopic simulation models. Then, the optimal timing plans for a number of scenarios with different geometries and traffic demand will be optimized using the TRANSYT-7F signal optimization programs utilizing the default and adjusted parameters of the program. The plans will be simulated in CORSIM, and the output performance measures will be computed and compared. The effect of parameter adjustment on the CORSIM assessment of the TRANSYT-7F optimization of signal timings will be examined.

\section{TRANSYT-7F Optimization Parameters}

All optimizations in TRANSYT-7F were performed using the minimization of the total delays as the optimization criteria. The optimized plans were implemented in CORSIM, and the delay was extracted. This provided insight regarding the consistency between the TRANSYT-7F and CORSIM simulations.

As mentioned above, initially TRANSYT-7F optimization was conducted utilizing the default values of the model. Then, these models were adjusted and the optimization was repeated. The TRANSYT-7F parameter adjustment was made to reflect the parameters of CORSIM micro-simulation as discussed later in this chapter. In this study, the sample size comprised 10 runs for all of the cases. 
The optimization and measure of the performance collection process is summarized in Figure 14.

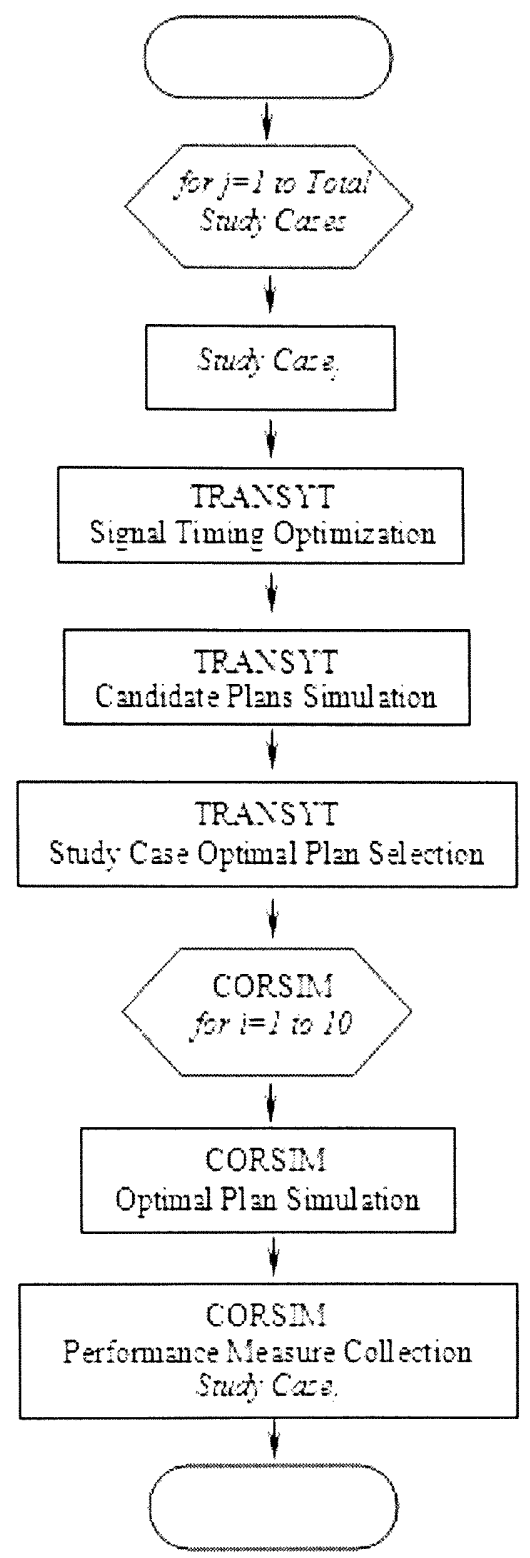

Figure 14: General Procedure for the Collection of the Measures of Performance 


\section{Case Study Description}

Four different systems were considered in this study: Systems A, B, C, and D. The systems were defined from the simplest to the most complex. The main parameters used to characterize the systems are network configuration, number of lanes, link lengths, and traffic demand. System A consists of a hypothetical, single, two-lane unidirectional link connecting two coordinated intersections. The link length between the two intersections was set as $250,500,1000$, and $2000 \mathrm{ft}$ to determine the effect of link lengths on the results. The volumes at the intersections were set up such that only under-saturated (volume/capacity $<1.0$ ) operations were covered. The volume/capacity ratios examined at the downstream signal are $0.8,0.9$, and 1.0. No turning movements were considered at this level of analysis. The detailed geometry of the system is shown in Figure 15.

System B is defined similarly to System A, with the addition of left and right turning upstream feeding links in addition to the through feeding link, to simulate more realistic conditions. System B consists of a hypothetical, single, two-lane unidirectional link connecting two coordinated intersections. The link length between the two intersections was $2000 \mathrm{ft}$. The volume/capacity ratio that was examined at the downstream signal was 1.0. Figure 16 shows the detailed geometry and operational set up for System B.

Finally, a real world network was be used to examine the effects of using more complex networks. In particular, modified versions of the arterial corridor US-1 between SW $136^{\text {th }}$ Street and SW $98^{\text {th }}$ Street in Miami, Florida were studied. A variation of this network, System C, was tested in which the traffic volumes were reduced such that all of the nodes 
considered have saturation degrees ranging between 0.7 and 0.98 (see Figure 17). Another variation of this network, System D, was generated by reducing the link lengths to examine the effects of link lengths on the analysis results (see Figure 18). In System C, the link lengths are the actual link lengths for US- 1 between SW $136^{\text {th }}$ Street and SW 98 Street. In System D, the link lengths from System C were modified to represent a more homogenous link length network. The number of lanes is two for Systems A and B, and three for Systems C and D. There are two intersections each for Systems A and B and seven intersections each for Systems C and D. The traffic demand is defined by the saturation degree at intersection B for Systems A and B. Saturation degrees from 0.7 to 1.0 were considered. For Systems C and D, the intersections had saturation degrees ranging from 0.8 to 0.98 . Figures 15 to 18 show the details of the geometry and the movements at each intersection.

\section{Parameter Adjustments}

Model parameter adjustment is defined in this study as the task of adjusting the parameters of an existing model to better correspond to a reference system. In this study, the TRANSYT-7F parameters were adjusted to produce platoon dispersion, saturation flow rate, and travel speed as close as possible to those assessed by CORSIM.

The saturation flow rates as assessed by CORSIM were measured from dynamic animation for subject approaches, when the demand was sufficient to measure these rates. 


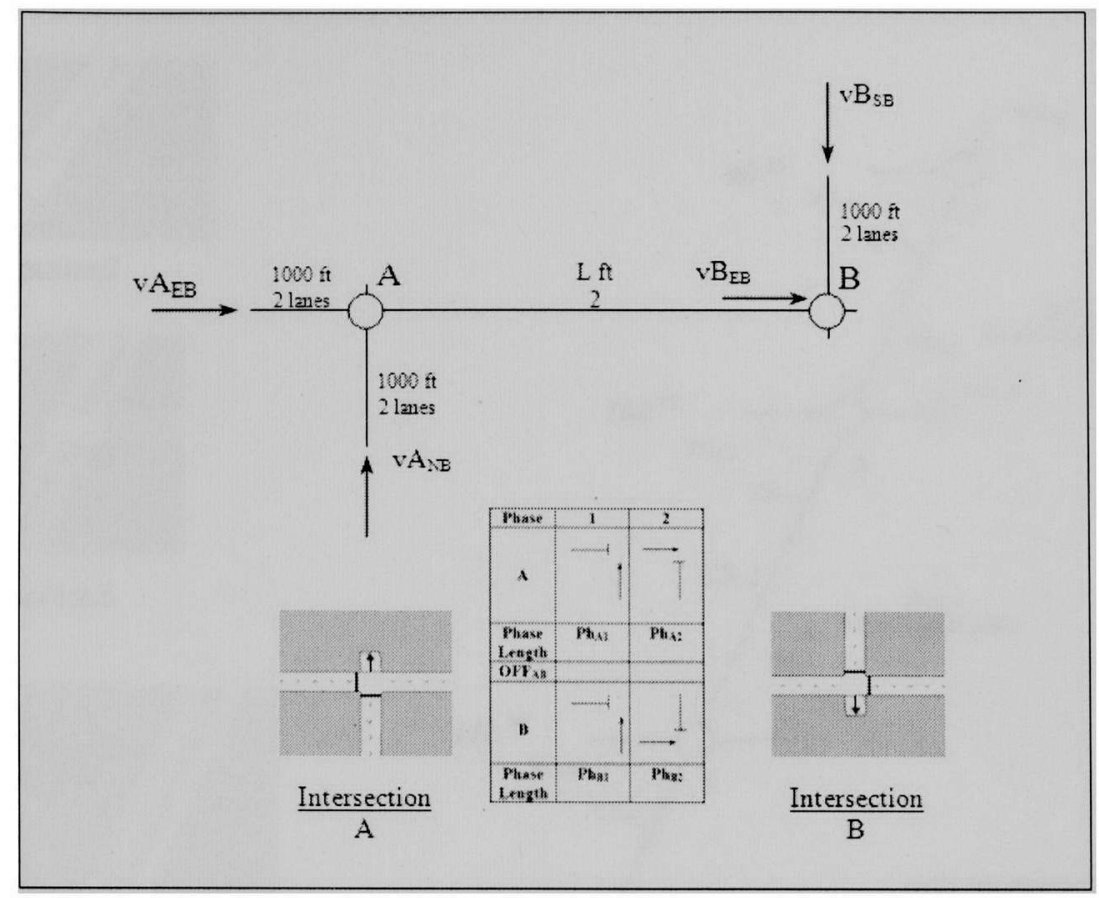

Figure 15: System A Geometry and Operational Set Up
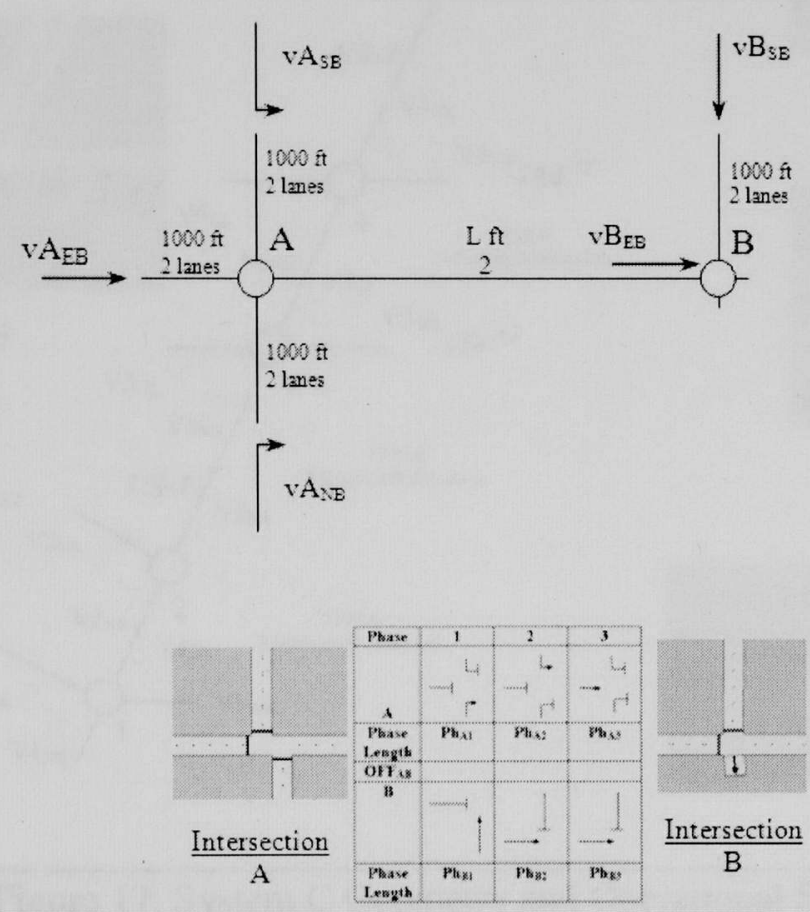

Figure 16: System B Geometry and Operational Set Up 


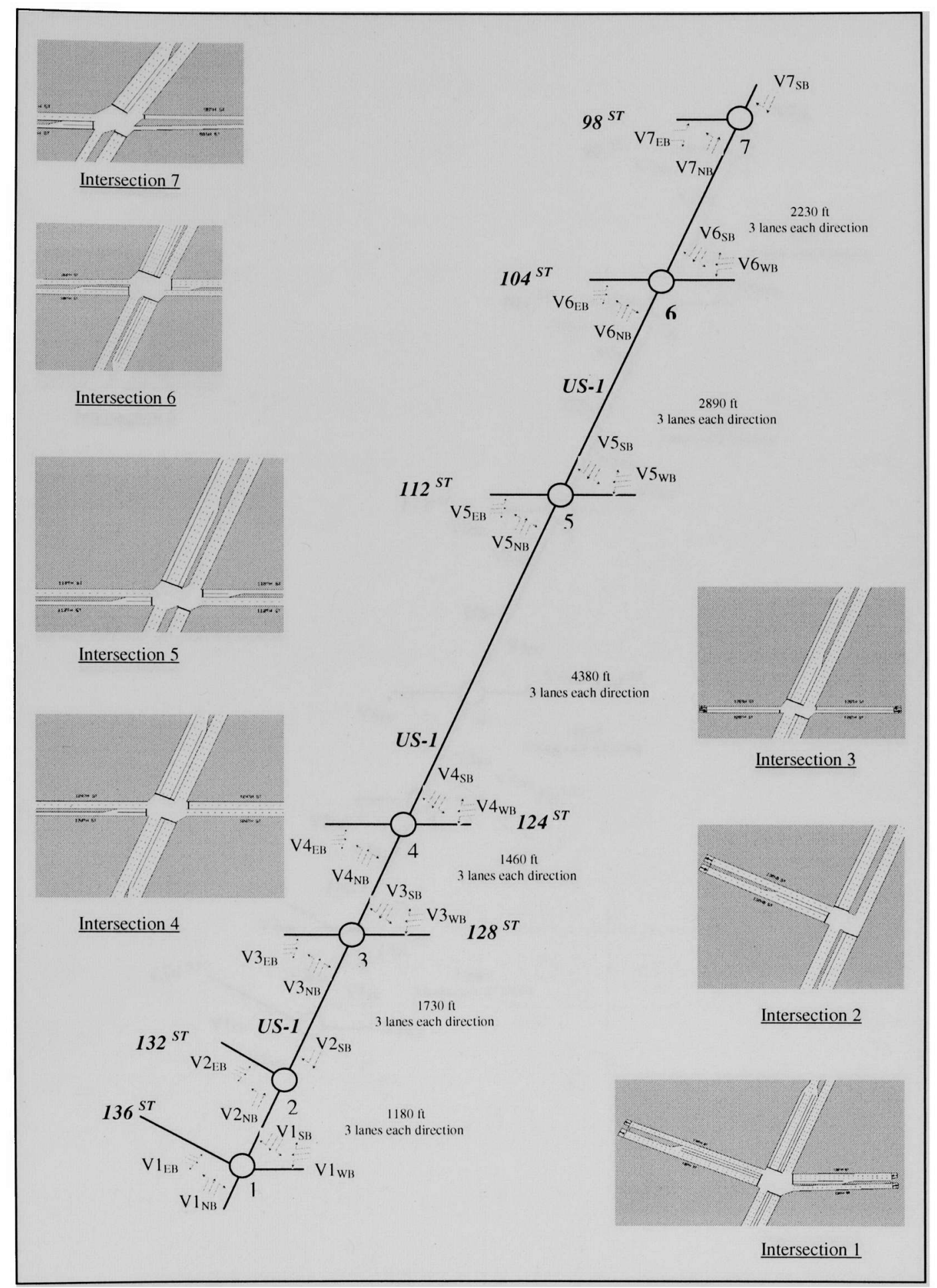

Figure 17: System C Geometry and Operational Set Up 


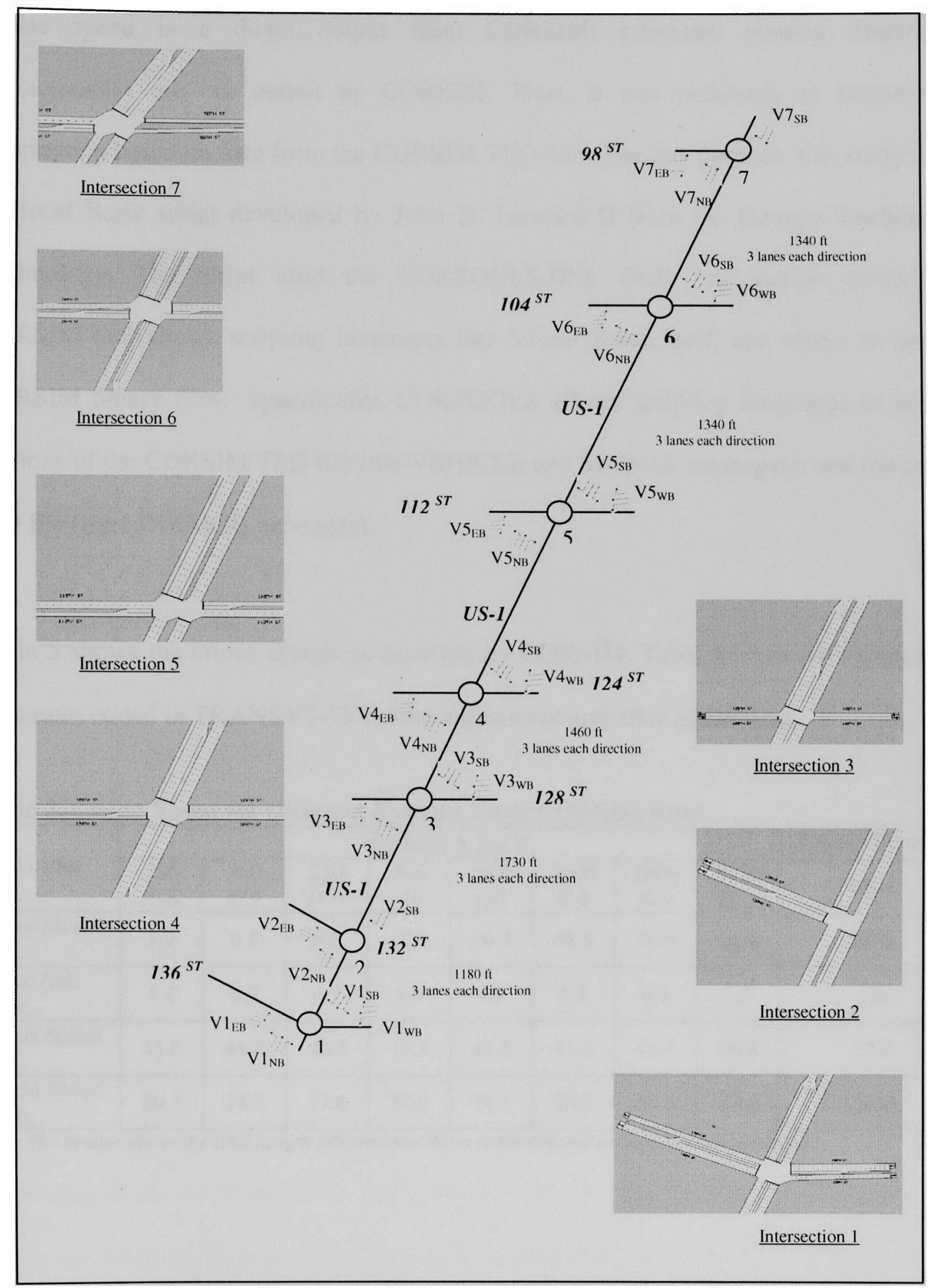

Figure 18: System D Geometry and Operational Set Up 
Cruise speed is a direct output from CORSIM; however, platoon dispersion characteristics are not output by CORSIM. Thus, it was necessary to obtain this information based on data from the CORSIM TSD files. For this purpose, this study used a Visual Basic script developed by John D. Leonard II from the Georgia Institute of Technology. The script used the CORTOOLS.DLL OLE "automation server" in CORSIM that allows scripting languages like Visual Basic, perl, and others to access CORSIM binary files. Specifically, CORTOOLS allows scripting languages to access portions of the CORSIM TSD file (the VEHICLE and SIGNAL messages), and the entire TID file (the LINKMOE messages).

Table 5 shows the cruise speeds as assessed by CORSIM. Table 6 shows the saturation flow rates coded in TRANSYT-7F before adjustment and after adjustment.

Table 5: Cruise Speed for Different Systems From CORSIM Runs

\begin{tabular}{|l|c|c|c|c|c|c|c|c|c|}
\hline \multirow{2}{*}{ Parameter } & \multicolumn{7}{|c|}{ Systems And B } & Systems C and D \\
\cline { 2 - 9 } & $\begin{array}{c}250 \\
/ 0.8\end{array}$ & $\begin{array}{c}250 \\
/ 0.9\end{array}$ & $\begin{array}{c}250 \\
11.0\end{array}$ & $\begin{array}{c}500 \\
/ 10\end{array}$ & $\begin{array}{c}1000 \\
/ 10\end{array}$ & $\begin{array}{c}2000 \\
/ 0.8\end{array}$ & $\begin{array}{c}2000 \\
/ 0.9\end{array}$ & $\begin{array}{c}2000 \\
/ 1.0\end{array}$ & US-1 \\
\hline $\begin{array}{l}\text { Time Mean } \\
\text { s }\end{array}$ & 5.9 & 6.1 & 6.3 & 12.4 & 24.4 & 48.5 & 46.0 & 49.8 & 18.9 \\
\hline $\begin{array}{l}\text { Time Std. } \\
\text { Dev. }\end{array}$ & 1.2 & 0.9 & 0.7 & 1.4 & 1.9 & 4.3 & 6.5 & 3.9 & 2.0 \\
\hline $\begin{array}{l}\text { Mean Speed } \\
\text { ft/s }\end{array}$ & 45.0 & 41.5 & 40.4 & 40.8 & 41.2 & 41.6 & 44.4 & 40.4 & 52.8 \\
\hline $\begin{array}{l}\text { Mean Speed } \\
\text { mph }\end{array}$ & 30.7 & 28.3 & 27.6 & 27.8 & 28.1 & 28.3 & 30.3 & 27.6 & 36.0 \\
\hline
\end{tabular}

Note: The header show's the Link Length ft/Saturation Ratio at the downstream intersection approach. 
Table 6: Default and Adjusted Time Headways and Saturation Flow Rates for Different Systems

\begin{tabular}{|c|c|c|c|c|c|c|c|c|c|}
\hline Scenario & & $\begin{array}{c}\text { System } \\
\text { A }\end{array}$ & \multicolumn{3}{|c|}{ System B } & \multicolumn{4}{|c|}{ Systems C and D } \\
\hline \multirow{4}{*}{$\begin{array}{l}\text { Parameters } \\
\text { form } \\
\text { CORSIM } \\
\text { used to } \\
\text { adjust } \\
\text { TRANSYT- } \\
\text { 7F }\end{array}$} & Parameter & Thru & Thru & $\begin{array}{l}\text { Dual } \\
\text { Right } \\
\text { Turn }\end{array}$ & $\begin{array}{l}\text { Dual } \\
\text { Left } \\
\text { Turn }\end{array}$ & Thru & $\begin{array}{l}\text { Single } \\
\text { Left } \\
\text { Turn }\end{array}$ & $\begin{array}{l}\text { Single } \\
\text { Right } \\
\text { Turn }\end{array}$ & $\begin{array}{l}\text { Dual } \\
\text { Left } \\
\text { Turn }\end{array}$ \\
\hline & $\begin{array}{c}\text { Time headway } \\
\text { s/veh }\end{array}$ & 0.58 & 0.58 & 0.49 & 0.46 & 0.48 & 0.50 & 0.43 & 0.50 \\
\hline & $\begin{array}{c}\text { Saturation } \\
\text { Flow Rate } \\
\text { veh/hr }\end{array}$ & 2088 & 2088 & 1764 & 1960 & 1728 & 1800 & 1548 & 1809 \\
\hline & $\begin{array}{c}\text { Time } \\
\text { Headway } \\
\text { Deviation } \\
\text { s/veh } \\
\end{array}$ & 0.06 & 0.06 & 0.08 & 0.08 & 0.07 & 0.10 & 0.09 & 0.07 \\
\hline \multirow{3}{*}{$\begin{array}{l}\text { Unadjusted } \\
\text { TRANSYT- } \\
\text { 7F } \\
\text { Parameters }\end{array}$} & & $\begin{array}{c}\text { System } \\
\text { A }\end{array}$ & \multicolumn{3}{|c|}{ System B } & \multicolumn{4}{|c|}{ Systems C and D } \\
\hline & Parameter & Thru & Thru & $\begin{array}{l}\text { Dual } \\
\text { Right } \\
\text { Turn } \\
\end{array}$ & $\begin{array}{l}\text { Dual } \\
\text { Left } \\
\text { Turn } \\
\end{array}$ & Thru & $\begin{array}{l}\text { Single } \\
\text { Left } \\
\text { Turn } \\
\end{array}$ & $\begin{array}{l}\text { Single } \\
\text { Right } \\
\text { Turn } \\
\end{array}$ & $\begin{array}{l}\text { Dual } \\
\text { Left } \\
\text { Turn } \\
\end{array}$ \\
\hline & $\begin{array}{c}\text { Saturation } \\
\text { Flow Rate } \\
\text { veh/hr }\end{array}$ & 1800 & 1800 & 1710 & 1620 & 1800 & 1620 & 1710 & 1620 \\
\hline
\end{tabular}

A successful application of Robertson's platoon dispersion model to modeling platoon dispersion relies in the appropriate adjustment of several model parameters. The empirical studies performed by the TRRL in the United Kingdom suggest some default values for platoon dispersion modeling. The work performed by PRC Engineering (1) and the University of Florida (1) suggested a set of default values for the platoon dispersion parameters for the North American version of TRANSYT-7F.

According to the TRANSYT-7F User's Manual, the following values could be used to obtain reasonable estimates for the Platoon Dispersion Factor (PDF). 
- The default value of 0.35 implies moderate friction [light turning traffic, light pedestrian traffic, 11- to 12 -ft or 3.3- to 3.6-meter lanes, possibly divided, and a well-designed Central Business District (CBD) or fringe arterial street].

- A value of 0.5 implies heavy friction (combination of parking, moderate to heavy turning traffic, moderate to heavy pedestrian traffic, narrow lane width, and traffic flow typical of an urban CBD).

- A value of 0.25 implies low friction (no parking, divided, turning provision $12-\mathrm{ft}$ or 3.6-meter lane width, and suburban high-type arterial street)

Although research findings (1) have indicated that the platoon dispersion parameters should be site-specific and be a function of the road grades, curvature, parking, opposing flow interference, traffic volume, and other sources of impedance, no formal methodology exists to calibrate the platoon dispersion parameter.

Regarding Robertson's platoon dispersion, Equation (1) suggests that the traffic flow, which arrives during a given interval at the downstream intersection of a link, is a weighted combination of the arrival pattern downstream of the link during the previous time interval and the departure pattern from the upstream traffic signal $\Delta t$ seconds ago. Further expansion of the recurrence dispersion equation by Seddom (11) took the following form:

$$
q_{t}=\sum_{i=T}^{\infty}\left[F(1-F)^{i-T}\right]_{t-i}
$$


Equation 3 illustrates that the link travel time in the TRANSYT-7F recurrence platoon dispersion model actually follows a shifted geometrics distribution (shifted $\mathrm{T}$ seconds to the right from the origin). In light of a generalized format of the platoon dispersion model, Yu and Van Aerde (12) examined the use of several other possible probability distributions, including normal distribution of link travel times, normal distribution of speeds, and exponential distribution of link travel times. They concluded that while the fundamental probability distributions are different in nature for a sample of upstream departure flows, the dispersion based on different distribution formats resulted in relatively similar downstream arrival patterns.

Applying the basic properties of a geometric distribution to Equation (4.3) will result in the following three equations:

$$
\beta=\frac{1}{1+\alpha}
$$

$$
\alpha=\frac{\sqrt{1+4 \sigma^{2}}-1}{2 t_{a}+1-\sqrt{1+4 \sigma^{2}}}
$$

$$
F=\frac{\sqrt{1+4 \sigma^{2}}-1}{2 \sigma^{2}}
$$

Where $\sigma$ is the standard deviation of the link travel times and $t_{a}$ is the average travel time. 
Equation (4.4) shows that the value of the travel time factor $\beta$ is dependent on the value of the platoon dispersion factor $\alpha$, as opposed to the fixed value of 0.8 , which is being used in TRANSYT-7F. Equation (4.5) illustrates that the value of $\alpha$ can be calibrated from the average link travel time and the respective standard deviation. Equation (4.6) further permits the calculation of the smoothing factor $F$ directly from the standard deviation of the of link travel times. In the TRANSYT-7F platoon dispersion model, the smoothing factor is computed based on a fixed value of the average link travel time.

TRANSYT-7F uses $F$ in its calculation of platoon dispersions. The values of $\alpha$ and $\beta$ are only used as an intermediate parameter for TRANSYT platoon dispersion. Therefore, it is possible to use the derived value of alpha based on the calibrated $F$ instead of the calibrated $\alpha$. To this end, to produce an F in TRANSYT-7F comparable to those in CORSIM, the value of $\alpha$ should be calculated inversely using 0.8 for $\beta$ (as is fixed in TRANSYT-7F). We will call this new value of alpha $\alpha_{F-b a s e d}$.

In this study, the F value was calculated based on the speed statistics from data extracted from the CORSIM TSD file. The calculated F value was used to calculate $\alpha_{\text {F-based }}$ using Equation (2.2), assuming that $\beta=0.8$. Table 7 shows a comparison of the $\alpha$ value obtained for different link lengths, as well as $\mathrm{V} / \mathrm{C}$ ratios from this study and from $\mathrm{Yu}$ and $\mathrm{Van}$ Aerde's study.

Generally speaking, it is observed that the lower the link length the higher the F value. Also, F shows a dependency on the value of the degree of saturation. This is expected 
because $\mathrm{F}$ is inversely proportional to travel time according to Equation (2). The lower the $\mathrm{V} / \mathrm{C}$ ratio the lower $\mathrm{F}$ is. Overall $\alpha_{\mathrm{F}-\mathrm{BASED}}$ does not change significantly over the lengths and the saturation degrees and ranges between 0.07 and 0.16 . As mentioned earlier, the recommended $\alpha$ value in TRANSYT-7F ranges from 0.25 to 0.5 depending on the friction level, and the default value is 0.35 . Low values of alpha correspond to low friction and minimal platoon dispersion.

Table 7: Robertson's Platoon Dispersion Parameters Different Sources

\begin{tabular}{|l|c|c|c|c|c|c|c|c|}
\hline Source & $\begin{array}{c}\text { Traffic } \\
\text { Conditions }\end{array}$ & $\begin{array}{c}\text { Link } \\
\text { Length }\end{array}$ & $\begin{array}{c}\text { Average } \\
\text { Travel } \\
\text { Time }\end{array}$ & $\begin{array}{c}\text { Travel } \\
\text { Time } \\
\text { Standard } \\
\text { deviation }\end{array}$ & $\begin{array}{c}\alpha \\
\text { Eq. } \\
(4.5)\end{array}$ & $\begin{array}{c}\beta \\
\text { Eq. } \\
(4.4)\end{array}$ & $\begin{array}{c}\text { Eq. } \\
(4.6)\end{array}$ & $\begin{array}{c}\alpha_{F-} \\
\text { based } \\
\text { Eq. } \\
(2.2)\end{array}$ \\
\hline $\begin{array}{l}\text { Yu \& Van } \\
\text { Aerde, based } \\
\text { on field } \\
\text { measuremets }\end{array}$ & Heavy Traffic & 1050 & 23.66 & 2.22 & 0.08 & 0.92 & 0.36 & 0.09 \\
\cline { 2 - 10 } & Heavy Traffic & 1837 & 40.50 & 4.85 & 0.12 & 0.89 & 0.19 & 0.14 \\
\cline { 2 - 10 } & $\mathrm{V} / \mathrm{C}=0.8$ & 250 & 5.91 & 1.24 & 0.16 & 0.86 & 0.55 & 0.18 \\
\cline { 2 - 10 } & $\mathrm{V} / \mathrm{C}=0.9$ & 250 & 6.14 & 0.89 & 0.09 & 0.91 & 0.66 & 0.11 \\
\cline { 2 - 10 } & $\mathrm{V} / \mathrm{C}=1.0$ & 250 & 6.26 & 0.71 & 0.06 & 0.94 & 0.73 & 0.07 \\
\cline { 2 - 10 } \begin{tabular}{l} 
Present \\
study based \\
on CORSIM \\
\cline { 2 - 10 } runs
\end{tabular} & $\mathrm{V} / \mathrm{C}=1.0$ & 500 & 12.41 & 1.44 & 0.09 & 0.92 & 0.49 & 0.10 \\
\cline { 2 - 9 } & $\mathrm{V} / \mathrm{C}=1.0$ & 1000 & 24.42 & 1.88 & 0.06 & 0.94 & 0.41 & 0.07 \\
\cline { 2 - 10 } & $\mathrm{V} / \mathrm{C}=0.8$ & 2000 & 48.47 & 4.29 & 0.09 & 0.92 & 0.21 & 0.10 \\
\cline { 2 - 10 } & $\mathrm{V} / \mathrm{C}=0.9$ & 2000 & 45.96 & 6.54 & 0.15 & 0.87 & 0.14 & 0.16 \\
\hline
\end{tabular}

Note: $\alpha_{\text {F-bused }}$ is calculated inversely using equation 2 , given the values of $F$ computed using equation $4, \beta=0.8$ and the average travel time for the link.

It appears based on our results that a value of $\alpha$ around 0.1 is more appropriate. Interestingly, there is a strong correspondence between the values reported by $\mathrm{Yu}$ and Van Aerde (12) based on field measurements and the values obtained using simulation in our study. This indicates that the default value proposed by the TRANSYT-7F User's Manual, 0.35 , does not correspond to the values that best capture platoon dispersion behavior. A value of 0.1 seems to be more appropriate based on CORSIM results and $\mathrm{Yu}$ 
and Van Aerde's results. This value was used in this study when adjusting TRANSYT-7F parameters.

\section{Assessment of Plan Performance}

When optimizing, TRANSYT-7F maximizes (or minimizes, depending on the objective function) an objective function called the performance index (PI). The objective function is selected by the user. The best objective function to use depends on the desired operational characteristics of the system under consideration. In this study, the PI used was the delay on the links.

For CORSIM, the measure of performance used to asses the quality of the timing plans was also the delay time. The delay time is defined as the difference between the total travel time and the moving time, it represents the time that vehicles are delayed if they cannot travel at the free-flow speeds. It combines the effect of signal control timing plus the interaction with other vehicles. In all of the cases, the reported values in this study correspond to the average over ten runs with different seed numbers to account for the stochastic nature of CORSIM. 


\section{RESULTS}

This section presents a discussion of the results obtained in this study.

\section{System A Results}

As described in the methodology section, the link length and degree of saturation were varied in System A to investigate how these variables affect the consistency between the results of TRANSYT-7F and CORSIM. Using the default parameter set in TRANSYT$7 F$, optimal timing plans were obtained for 12 different scenarios, and those optimal timing plans were later simulated in CORSIM. The investigated link lengths between the upstream and downstream intersections were $250 \mathrm{ft}, 500 \mathrm{ft}, 1000 \mathrm{ft}$, and $2000 \mathrm{ft}$, and the degree of saturation of traffic arriving at the downstream intersection was $0.8,0.9$, and 1.0. The average resulting delay in s/veh for each case is shown in Figures 19 through 22. To quantify the difference between the results of TRANSYT-7F and CORSIM, the relative difference between the outcomes of the two programs for each scenario was computed. The results are shown in Figures 23 through 26. Figure 27 shows the average error for each length computed over the three saturation degrees. Figure 28 shows the average error for each saturation degree averaged over all link lengths. 


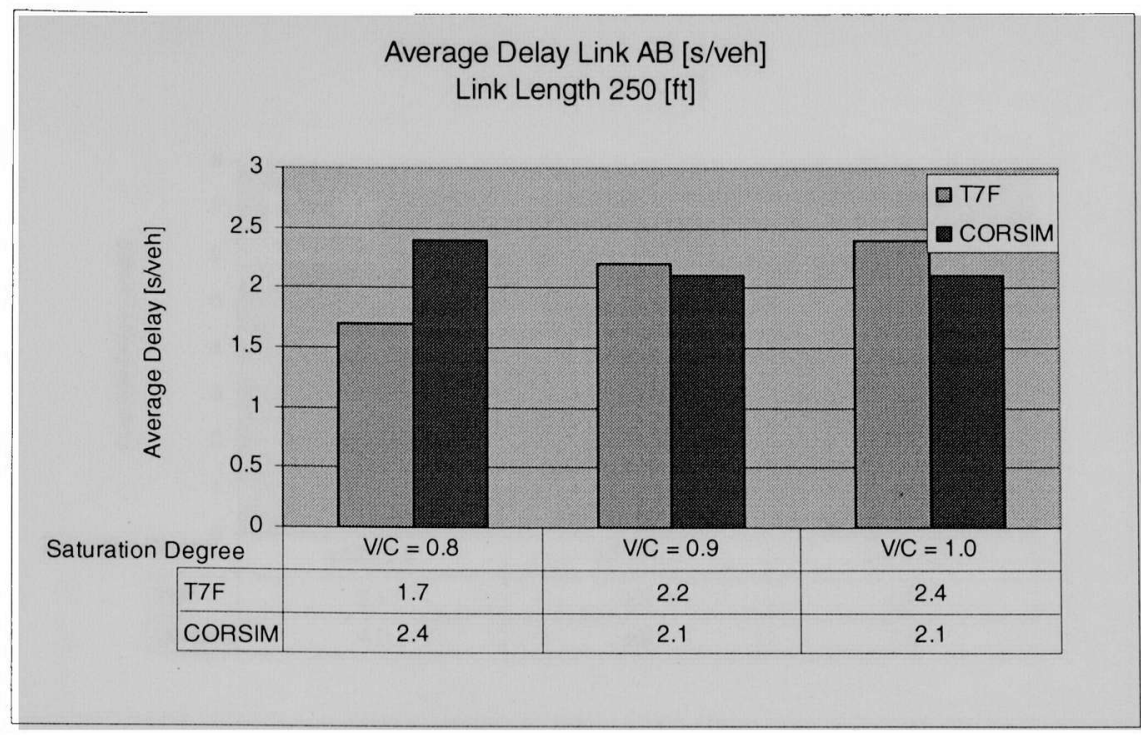

Figure 19: Comparison of TRANSYT-7F and CORSIM Delay Estimates, Link Length $250 \mathrm{ft}$

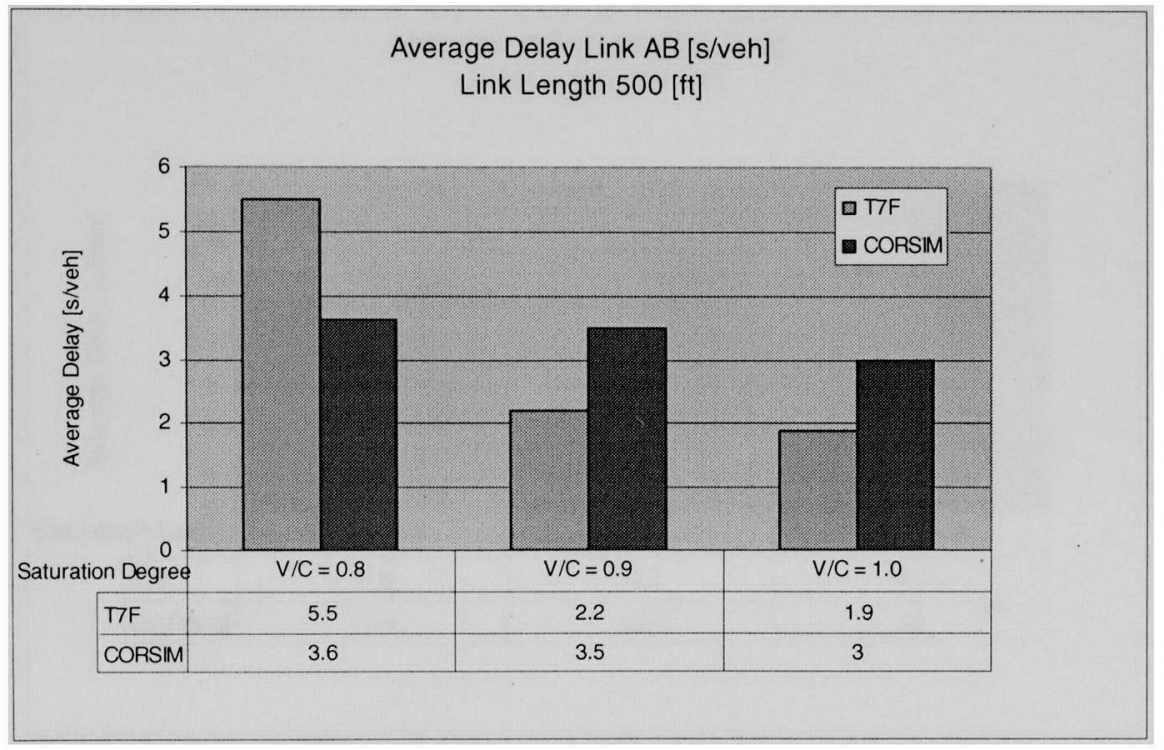

Figure 20: Comparison of TRANSYT-7F and CORSIM Delay Estimates, Link Length $500 \mathrm{ft}$ 


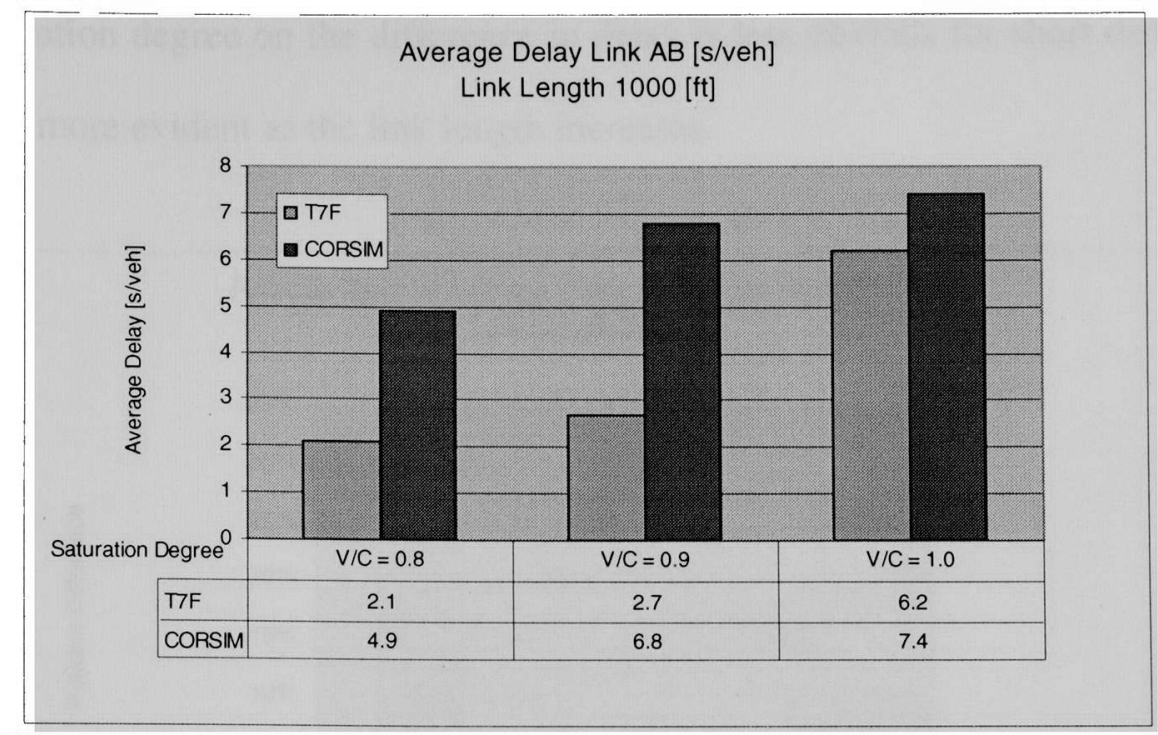

Figure 21: Comparison of TRANSYT-7F and CORSIM Delay Estimates, Link Length $1000 \mathrm{ft}$

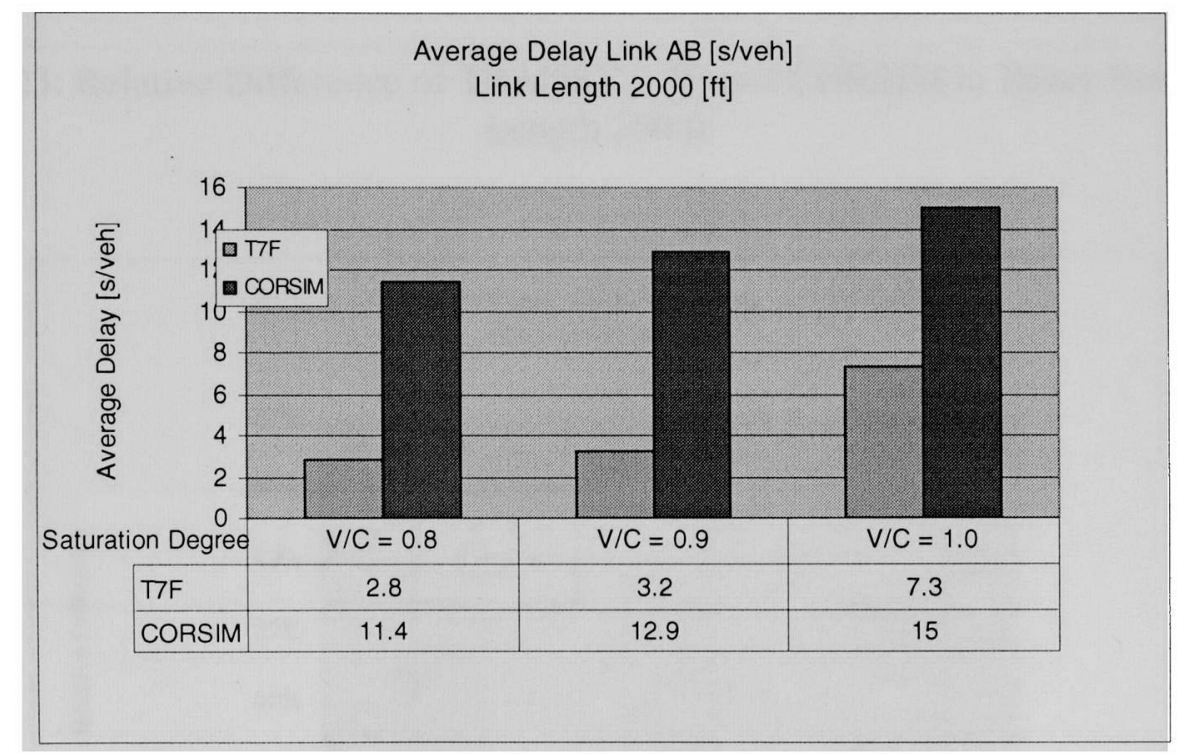

Figure 22: Comparison of TRANSYT-7F and CORSIM Delay Estimates, Link Length $2000 \mathrm{ft}$

From Figures 19 through 28, it is evident that the difference in the assessed values between TRANSYT-7F and CORSIM increased as the link length increased. The effect 
of the saturation degree on the difference in delay is less obvious for short distances and tends to be more evident as the link length increases.

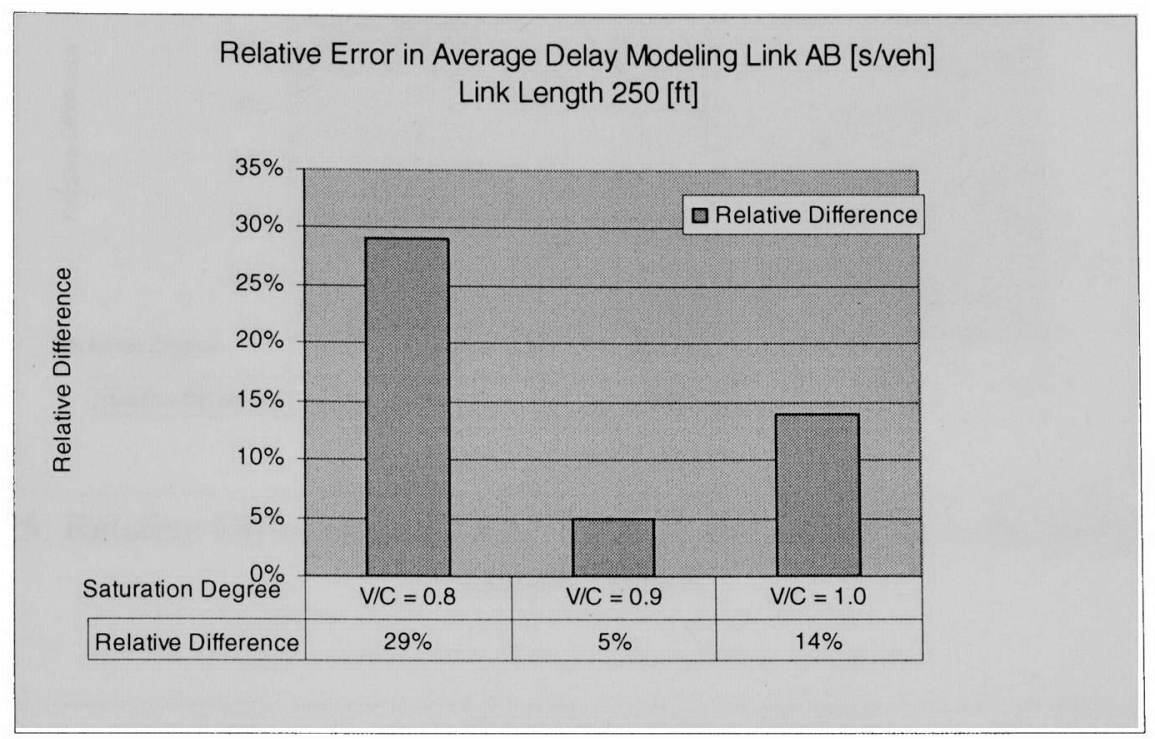

Figure 23: Relative Difference of TRANSYT-7F and CORSIM in Delay Estimation, Length $250 \mathrm{ft}$

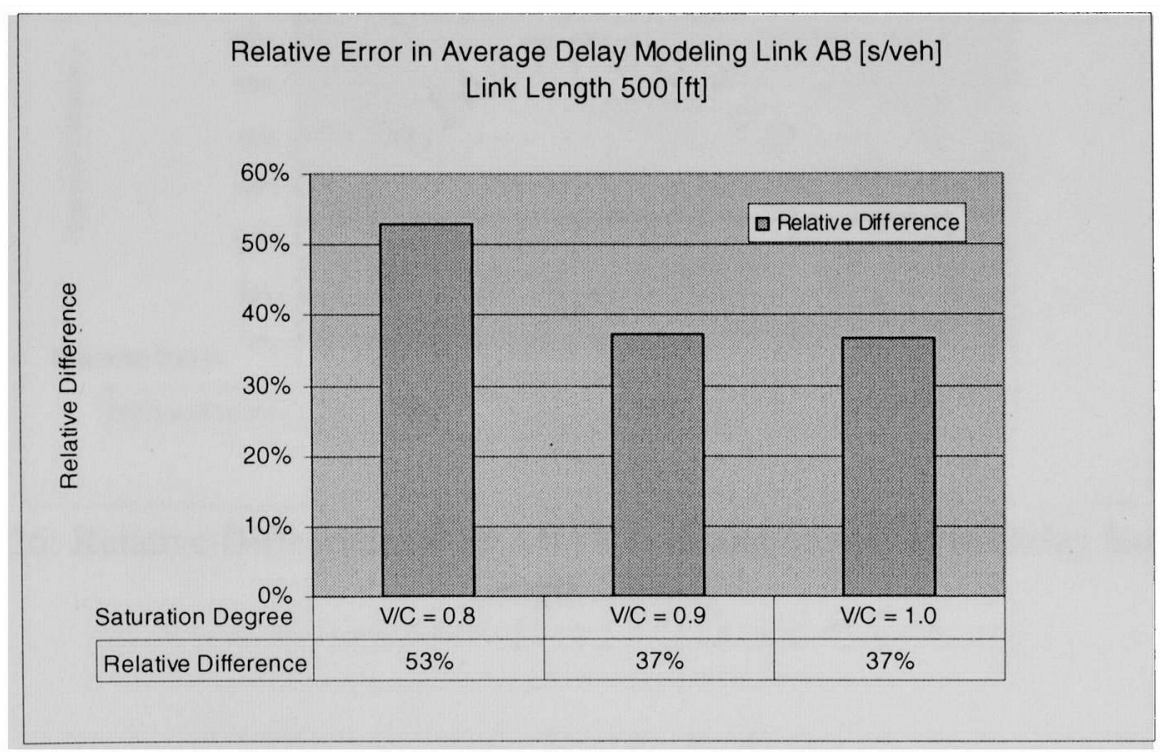

Figure 24: Relative Difference of TRANSYT-7F and CORSIM in Delay Estimation, Length $500 \mathrm{ft}$ 


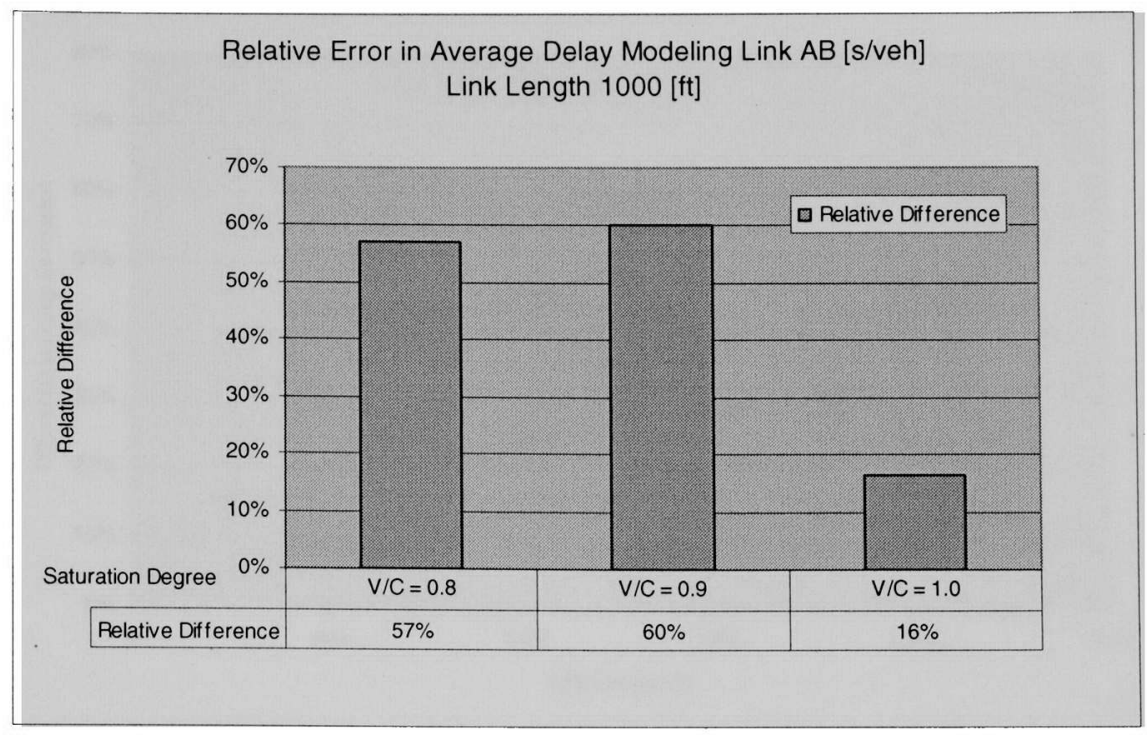

Figure 25: Relative Difference of TRANSYT-7F and CORSIM in Delay Estimation, Length $1000 \mathrm{ft}$

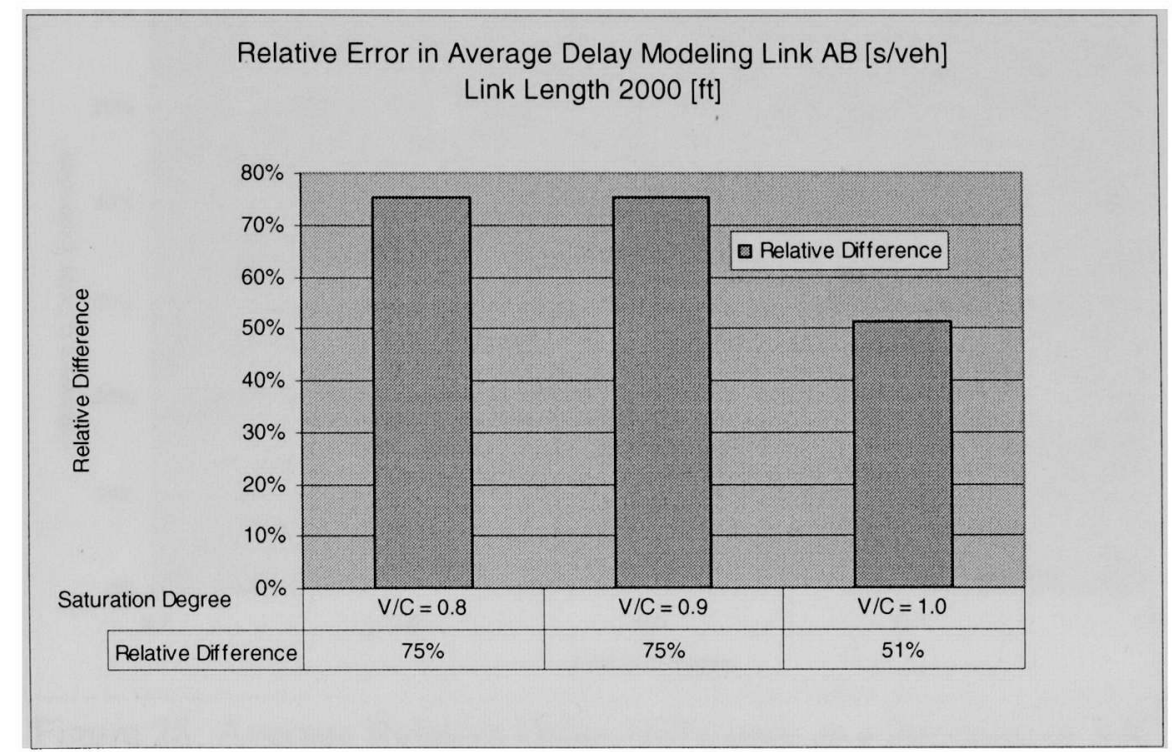

Figure 26: Relative Difference of TRANSYT-7F and CORSIM in Delay Estimation, Length $2000 \mathrm{ft}$ 


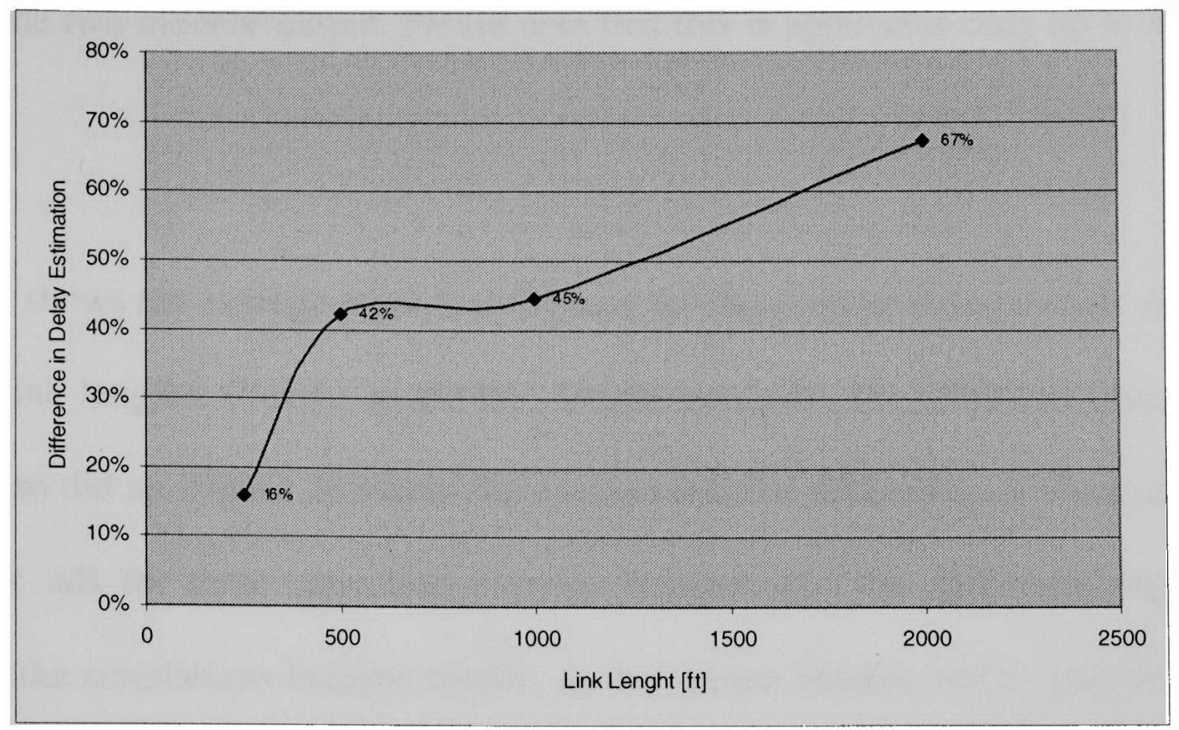

Figure 27: Average Relative Delay Difference as a Function of Link Length.

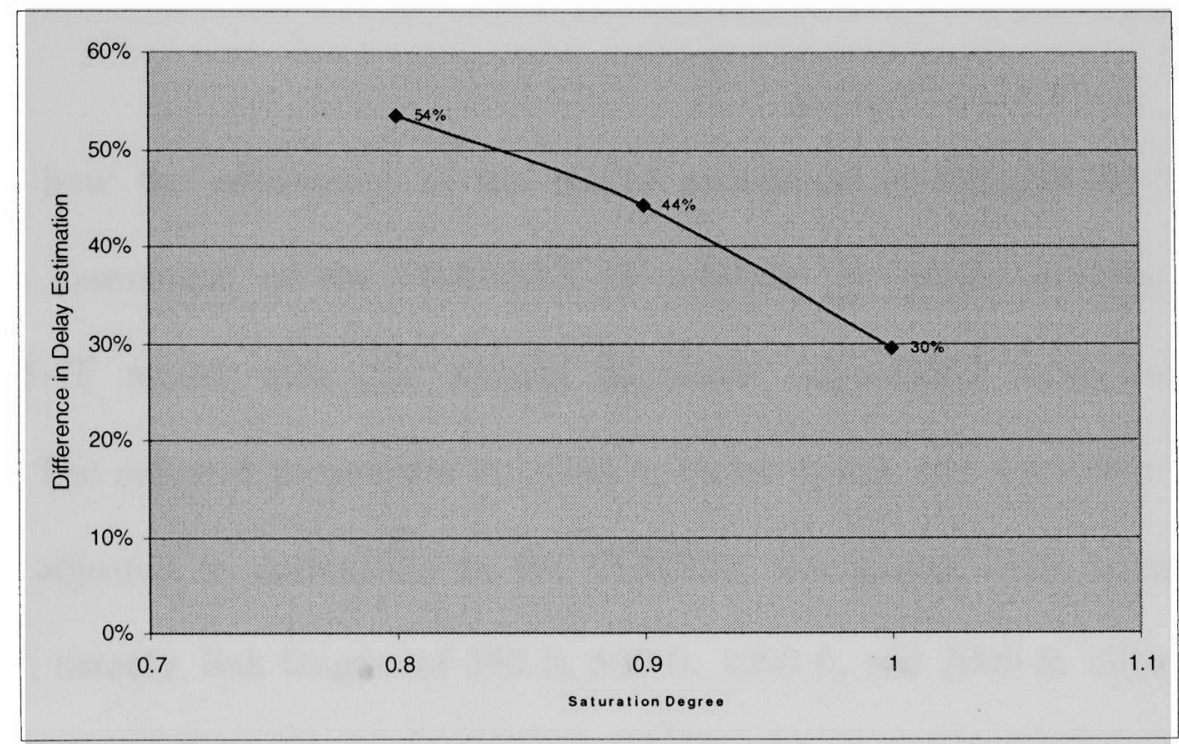

Figure 28: Average Relative Delay Difference as a Function of V/C.

From Figure 27, it can be inferred that the longer the link the higher the difference between TRANSYT-7F and CORSIM models in delay prediction. In addition, from Figure 28, it can be seen that the more congested the link, the smaller the difference 
between the two models' output. Please note that this is applicable only up to a V/C ratio of 1.0 .

Figure 27 shows the average relative difference for the average delay on link $\mathrm{AB}$ for four possible link lengths. Clearly, as the link length increased, the differences between both models also did so. Figure 28 shows the average relative differences in the average delay along link $\mathrm{AB}$ for three saturation degrees. Interestingly, the differences between the results of the simulations became smaller as the system became more congested (up to a $\mathrm{V} / \mathrm{C}=1.0$ ). This reflects the fact that the arrival patterns became more uniform across the time steps as traffic became heavier.

To assess how the adjustment of the model parameters in TRANSYT-7F impacts CORSIM's assessment of the TRANSYT-7F solution, the plans resulting from the TRANSYT-7F model with and without parameter adjustments were evaluated in CORSIM. The adjusted parameters included $\alpha$, cruise speed, and saturation flow rates that were adjusted to correspond to the CORSIM assessment. Four scenarios were considered, namely, link lengths of $250 \mathrm{ft}, 500 \mathrm{ft}, 1000 \mathrm{ft}$, and $2000 \mathrm{ft}$, all with $\mathrm{X}=1.0$. Figure 29 shows the percentage improvement in delays derived from using plans optimized with TRANSYT-7F with adjusted parameters compared to plans optimized using TRANSYT-7F without parameter adjustments. 
Figure 29 shows that, while the new plans lead to slightly better performances for $250 \mathrm{ft}$, $500 \mathrm{ft}$, and $1000 \mathrm{ft}$, for a link length of $2000 \mathrm{ft}$ the new timing plan performed worse than that obtained using TRANSYT-7F with the default parameter set.

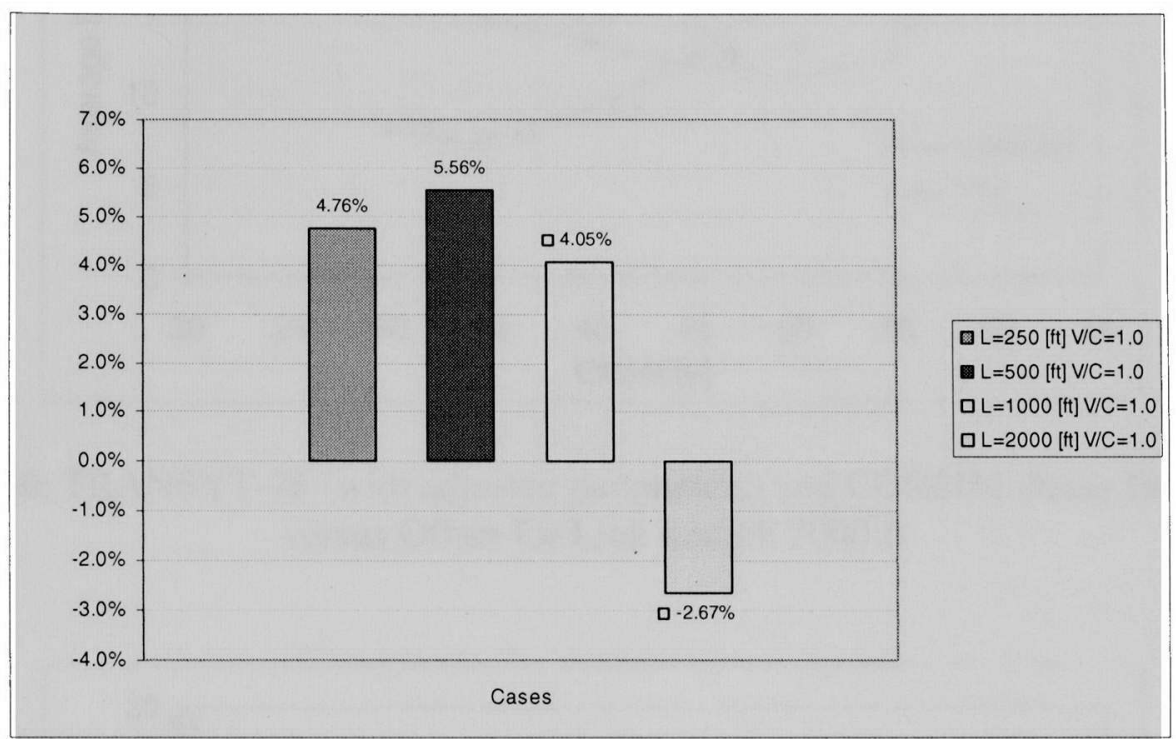

Figure 29: CORSIM Delay Improvement Estimate all Parameters Adjusted in TRANSYT-7F

Based on the above results, there is a need to answer the question as to why the benefits of TRANSYT-7F parameters had limited benefits according to CORSIM. To explore this, we explicitly compared the surfaces over the TRANSYT-7F search for the optimal signal timing plan and the actual representation in CORSIM of the same surface. Four cases were analyzed, with link lengths of $250 \mathrm{ft}, 500 \mathrm{ft}, 1000 \mathrm{ft}$, and $2000 \mathrm{ft}$, all with $\mathrm{V} / \mathrm{C}=1.0$. In particular, the offset was taken as the optimization variable, and the minimization of average link delay was assumed to be the target function. (Actually, in TRANSYT-7F, the target function is the delay in the system, but there is a strong correlation between this and the link average delay.) The results are given in Figures 30 through 33 . 


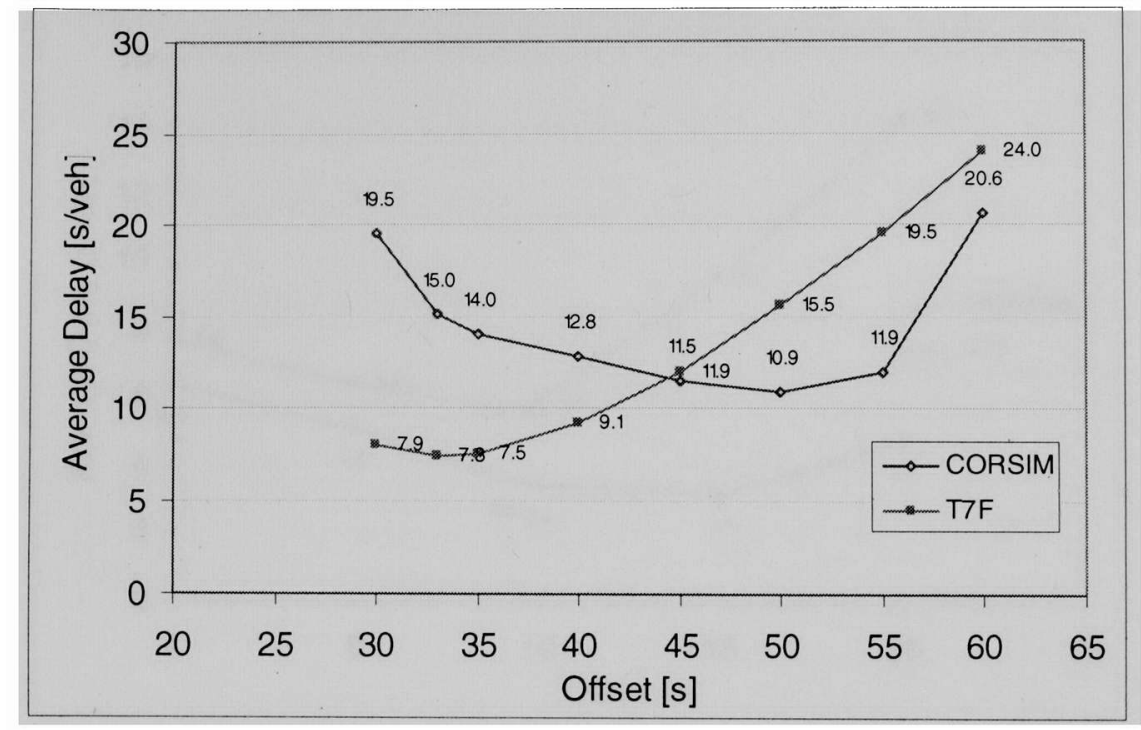

Figure 30: TRANSYT-7F (with adjusted parameters) and CORSIM Delay Estimation versus Offset for Link Length $2000 \mathrm{ft}$

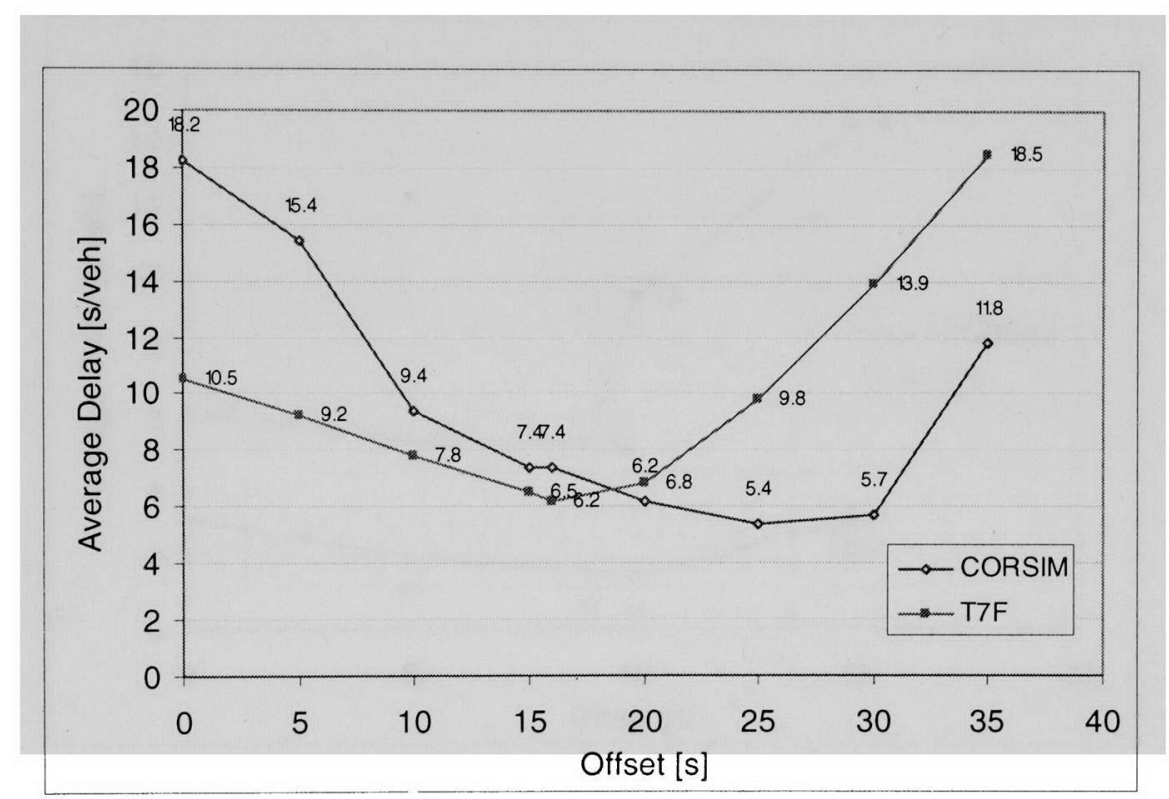

Figure 31: TRANSYT-7F (with adjusted parameters) and CORSIM Delay Estimation versus Offset for Link Length $1000 \mathrm{ft}$ 


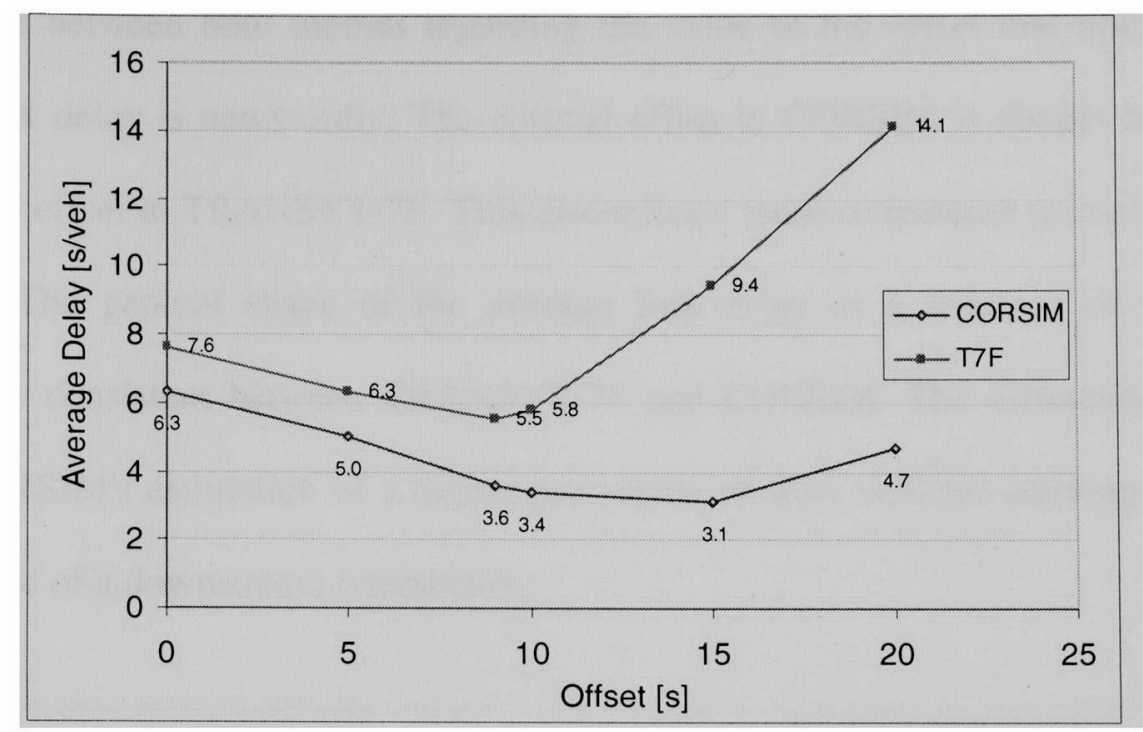

Figure 32: TRANSYT-7F (with adjusted parameters) and CORSIM Delay Estimation versus Offset for Link Length $500 \mathrm{ft}$

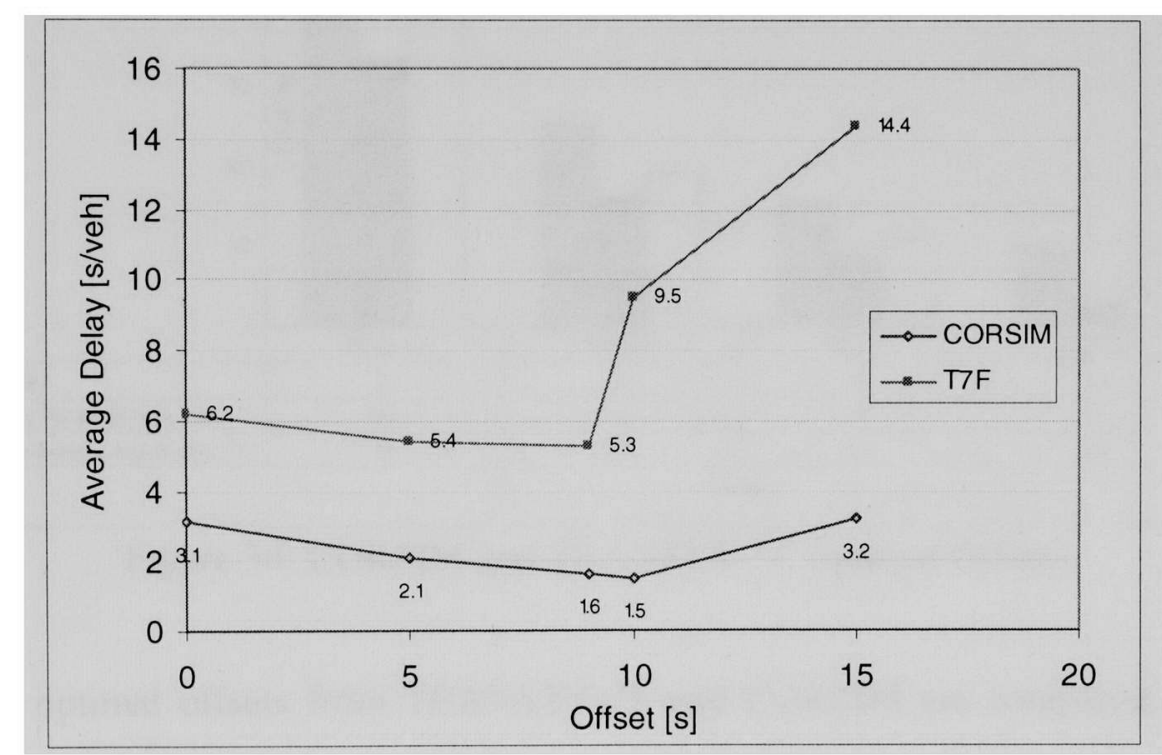

Figure 33: TRANSYT-7F (with adjusted parameters) and CORSIM Delay Estimation Versus Offset for Link Length $250 \mathrm{ft}$

The figures show the average link delay as a function of the offset between the upstream location and the intersections. Although there are differences in the average link delay that can be attributed to inherent differences in how both models compute this index, the 
discordance between both models regarding the value of the offset that minimizes the average link delay is noteworthy. The optimal offset in CORSIM is always higher than the optimal offset in TRANSYT-7F. This discordance tends to increase as the link length increases. The general shape of the average link delay as a function of the offset, however, is consistent between TRANSYT-7F and CORSIM. The difference could be due to CORSIM's estimation of a higher proportion of slow vehicles arriving late in the green period of a downstream intersection.

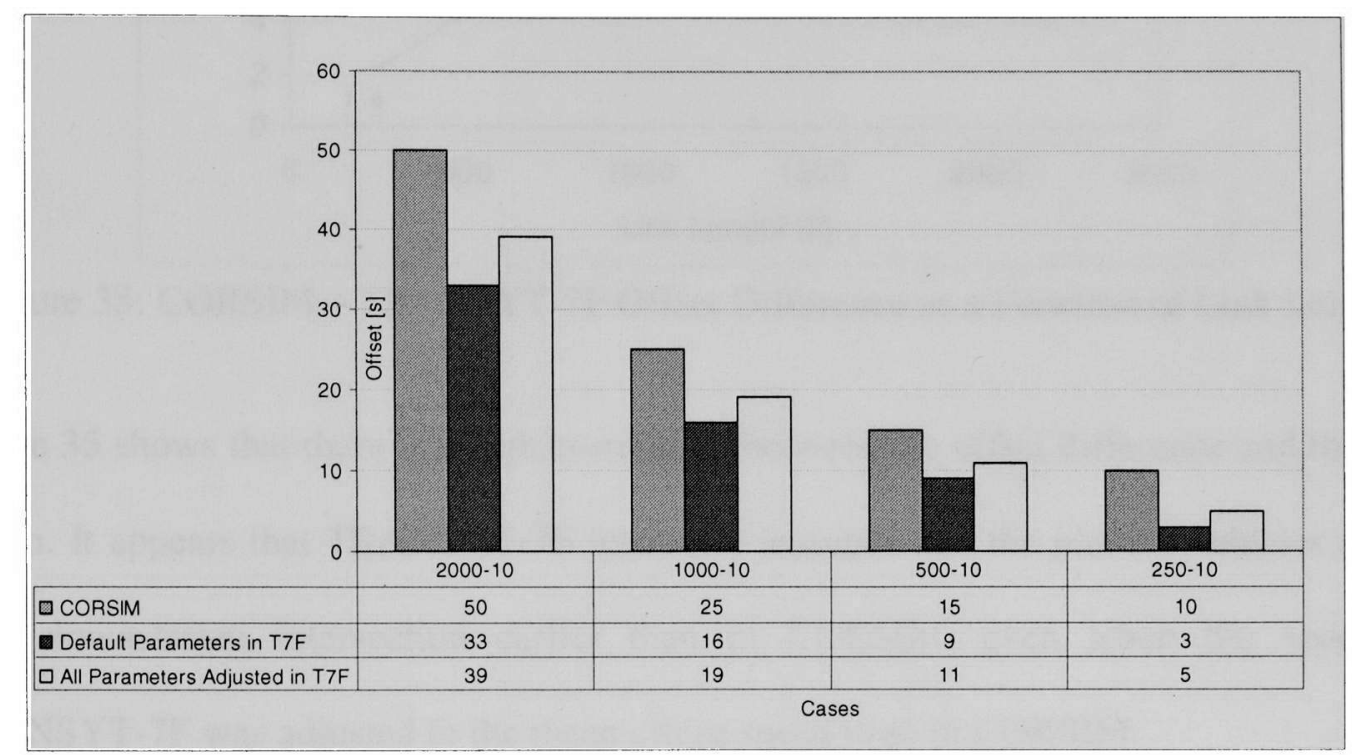

Figure 34: CORSIM and TRANSYT-7F Optimal Offsets

When the optimal offsets from TRANSYT-7F and CORSIM are compared, Figure 34 shows that adjusting the $\alpha$ value and the speed to reflect the average speed according to CORSIM helped move the TRANSYT -7F solution in the right direction (closer to the CORSIM solution). However, this was not sufficient to eliminate the inconsistencies between TRANSYT-7F and CORSIM. Figure 35 shows that the difference in the optimal offset according to TRANSYT-7F and CORSIM is generally a linear function of the link 
length. This explains why the TRANSYT-7F solution appeared to be worse for longer links

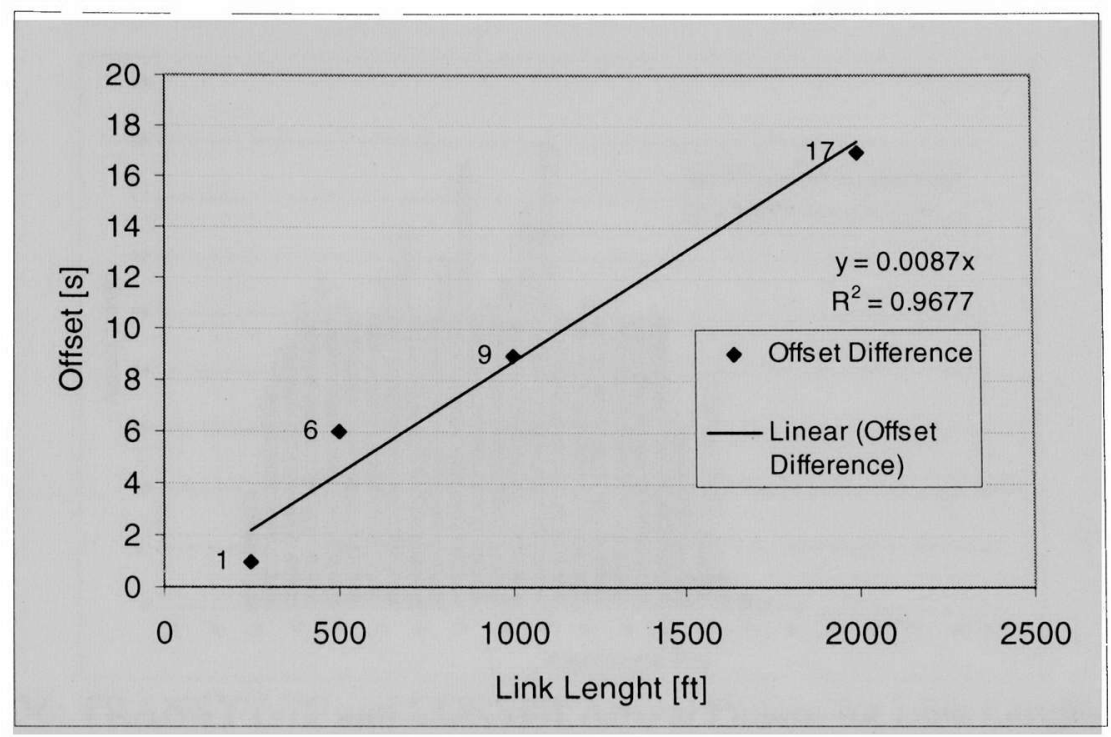

Figure 35: CORSIM - TRANSYT-7F Offset Difference as a Function of Link Length

Figure 35 shows that there is a high correlation between the offset difference and the link length. It appears that TRANSYT-7F internally assumes that the platoon vehicles arrive at a downstream intersection earlier than in CORSIM, even when the speed in TRANSYT-7F was adjusted to the mean cruise speed used in CORSIM.

To confirm the above results, the arrival patterns were extracted from TRANSYT-7F and CORSIM for link lengths $1000 \mathrm{ft}$ and $2000 \mathrm{ft}$ and V/C ratio of 1.0. The results are shown in Figures 36 and 37.

Figures 36 and 37 show that TRANSYT-7F tended to make the first vehicles arrive before their arrival in CORSIM, even when the cruise speed in TRANSYT-7F was 
adjusted to reflect the speeds from CORSIM. In addition, higher proportions of vehicles arrived at the latter part of the green in CORSIM.

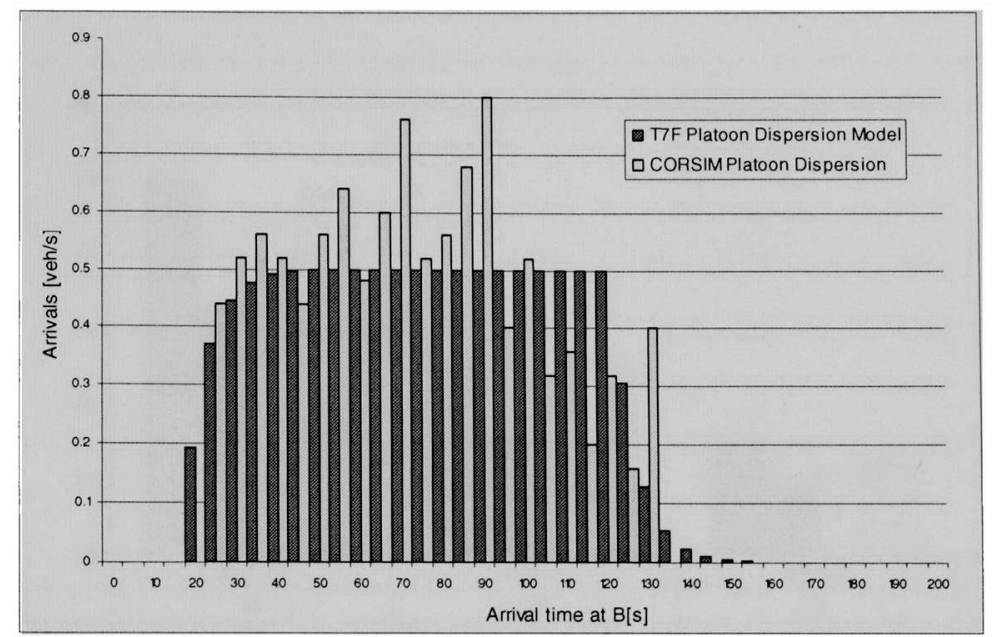

Figure 36: TRANSYT-7F and CORSIM Arrival Pattern for Link Length $1000 \mathrm{ft}$

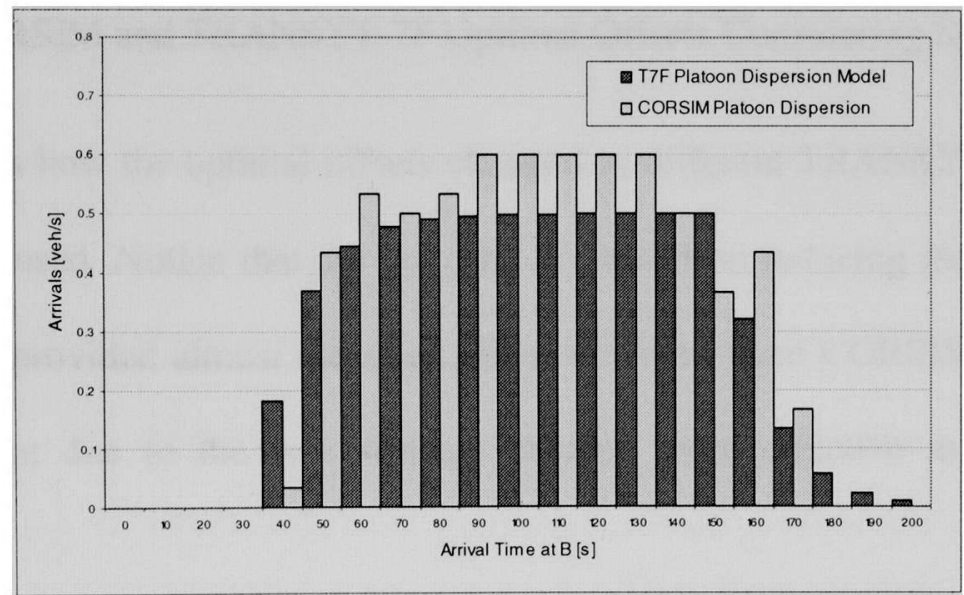

Figure 37: TRANSYT-7F and CORSIM Arrival Pattern for Link Length $2000 \mathrm{ft}$

A new optimization was conducted given the lessons just learned. Using four scenarios, link lengths $250 \mathrm{ft}, 500 \mathrm{ft}, 1000 \mathrm{ft}$, and $2000 \mathrm{ft}$, all with saturation degrees $\mathrm{V} / \mathrm{C}=1.0$, a new optimization procedure was carried out in TRANSYT-7F that considered further adjustments of speed. Instead of coding the average speed from CORSIM in TRANSYT- 
7F, the coded speed was $80 \%$ lower than the average speed from CORSIM. We will call this scenario "the speed corrected." The offsets and the errors relative to the optimal offset obtained from CORSIM are shown in Figures 38 and 39, respectively.

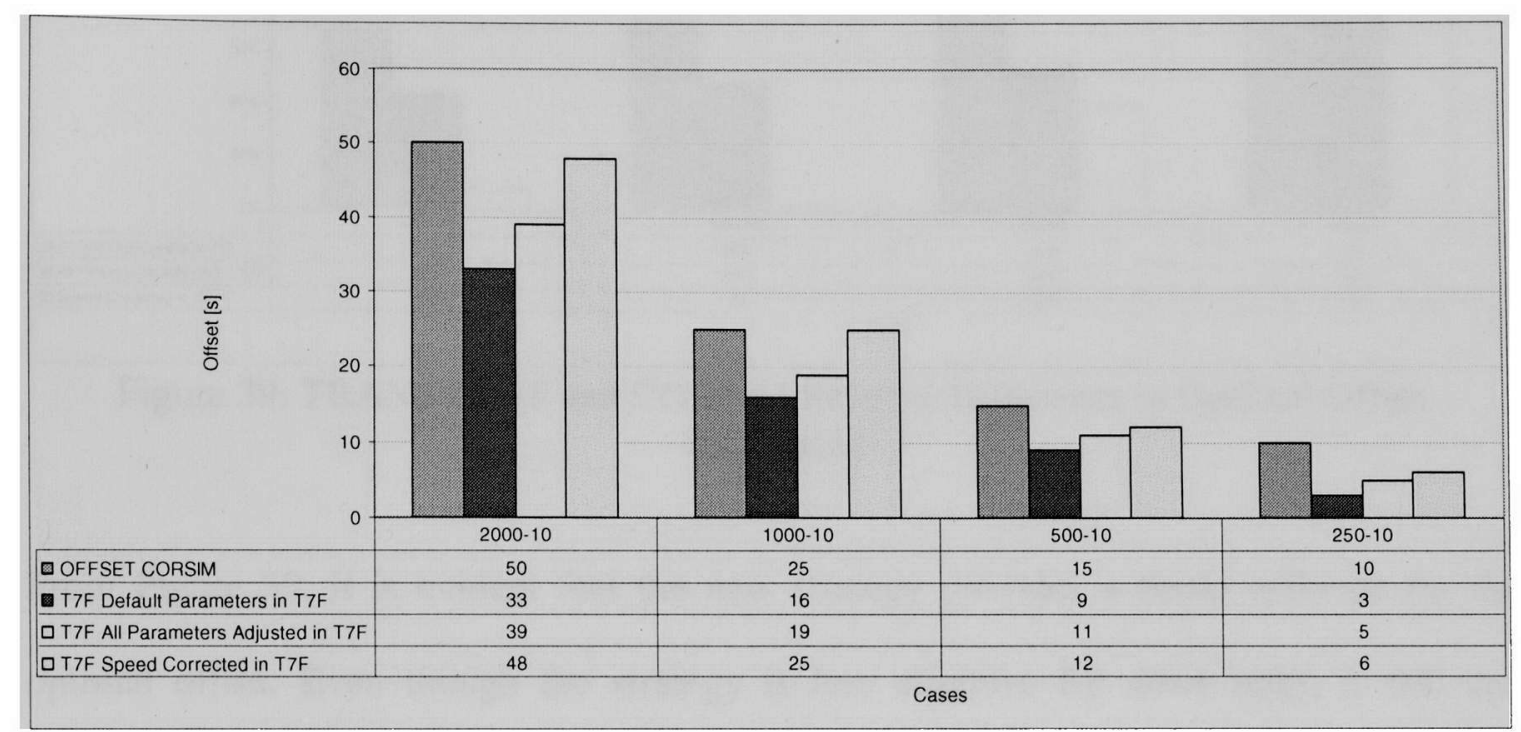

Figure 38: CORSIM and TRANSYT-7F Optimal Offsets Considering Speed Correction

Figure 38 shows how the optimal offsets changed as different TRANSYT-7F adjustment strategies were used. Notice that the last strategy based on reducing the coded speed in TRANSYT-7F provided almost the same offset observed form CORSIM. Also note that the improvement due to the new strategy became more effective as the link length became longer. 


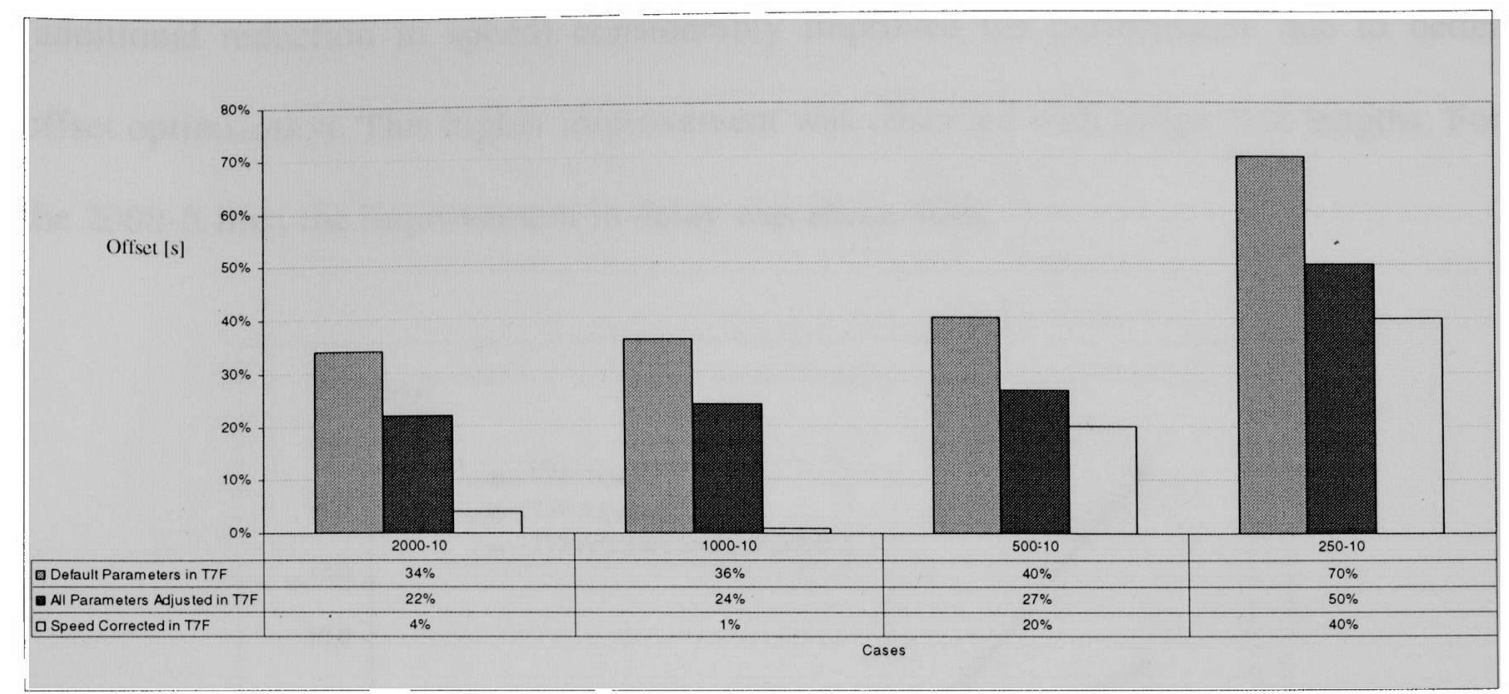

Figure 39: TRANSYT-7F and CORSIM Relative Difference in Optimal Offset Estimation

From Figure 39, it is evident that the new strategy provides a better estimate for the optimal offset. Even though the strategy is less effective for short links, it still can provide a reduction in the difference between CORSIM and TRANSYT-7F of as much as $30 \%$.

To finalize this exploratory process, a final optimization was conducted to test the behavior of the TRANSYT-7F solution and CORSIM assessment when the latter adjustment strategy is considered. Four scenarios were used, namely, link lengths $250 \mathrm{ft}$, $500 \mathrm{ft}, 1000 \mathrm{ft}$ and $2000 \mathrm{ft}$, all with saturation degrees $\mathrm{V} / \mathrm{C}=1.0$. The results are given in Figure 40.

From Figure 40, it can be observed that, according to CORSIM, no improvement in the performance of the timing plans was obtained from the use of TRANSYT-7F with adjusted parameters ( $\alpha$, speed and saturation flow). However, the correct strategy 
(additional reduction in speed) considerably improved the performance due to better offset optimization. This higher improvement was observed with longer link lengths. For the 2000 -ft link, the improvement in delay was about $40 \%$.

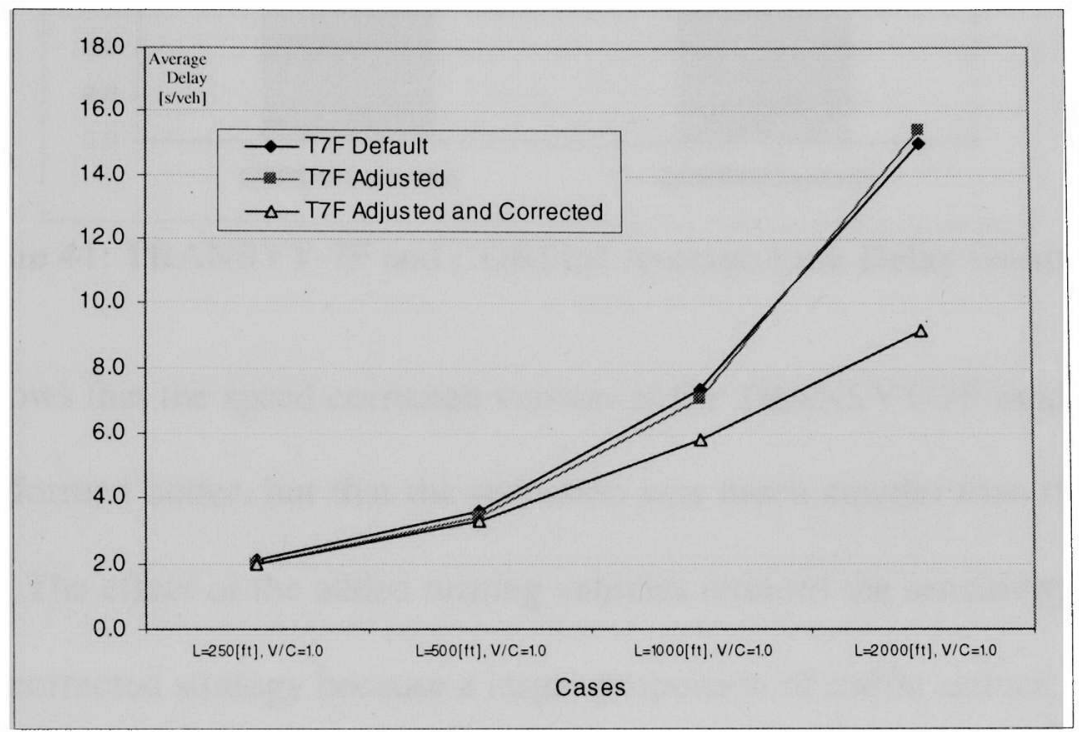

Figure 40: CORSIM Delay Estimate for Different Adjustment Strategies

\section{System B Results}

System B is a simple system with three feeder links contributing to the studied downstream link. System B is a modification of System A that includes additional feeder links to the studied link to investigate if the interaction between platoons from different feeder links affects the results. Using the calibrated and corrected parameter sets in TRANSYT-7F for System B, optimal timing plans were obtained for one scenario for later simulation in CORSIM. This scenario included a distance between intersections A and $\mathrm{B}$ of $2000 \mathrm{ft}$, and a V/C ratio of the studied link of 1.0. The average delay in s/veh for each case is shown in Figure 41. Also, the offset consistence was verified by comparing the optimal offsets from TRANSYT-7F and CORSIM; the results are shown in Figure 42. 


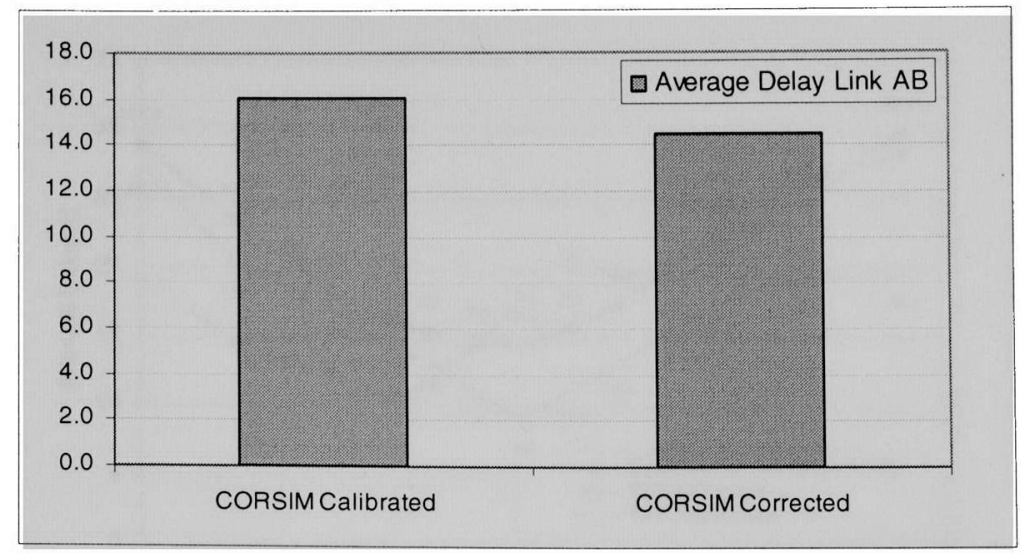

Figure 41: TRANSYT-7F and CORSIM Average Link Delay Estimation

Figure 41 shows that the speed corrected version of the TRANSYT-7F model produced a plan that performed better, but that the reduction was much smaller than those observed in System A. The effect of the added turning vehicles reduced the sensitivity of the model to the speed corrected strategy because a larger proportion of traffic arrived.

Figure 42 clearly shows how the effect of the speed correction switched the curve such that it now fits the assessment derived from CORSIM.

\section{Systems C and D Results}

Finally, we determined to what extent the findings obtained earlier also apply to a real world, more complex system. For this purpose a section of the US-1 corridor between SW $136^{\text {th }}$ Street and SW $98^{\text {th }}$ Street in Miami, Florida was considered. A detailed description of the corridor is presented in the case description section of this thesis. 


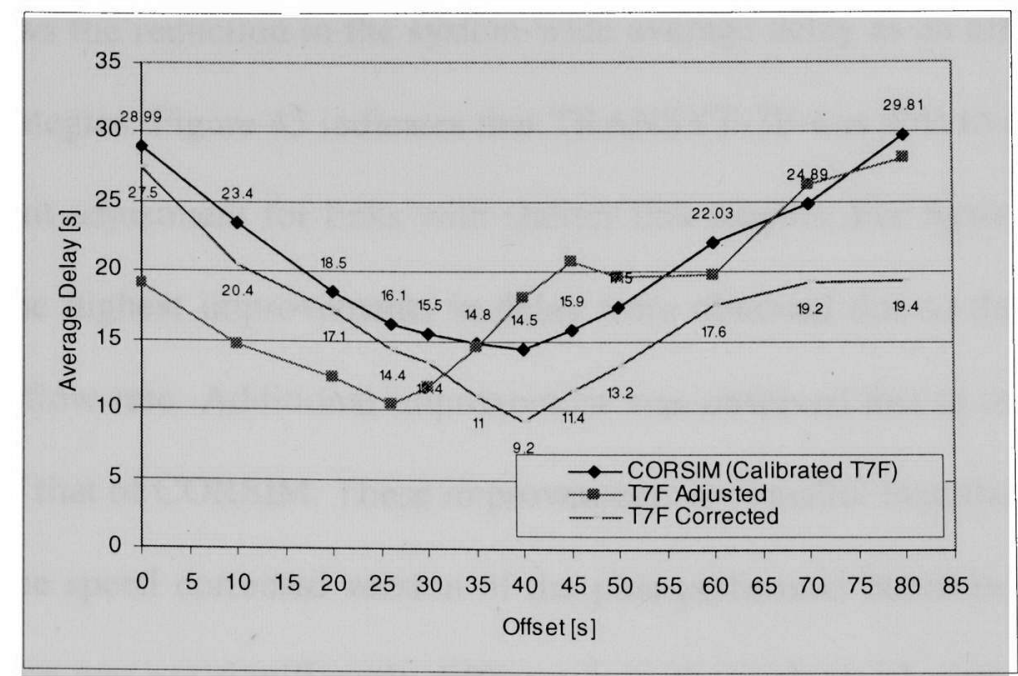

Figure 42: CORSIM and TRANSYT-7F Delay Estimation versus Offset in the System

Because one of the requirements of this study was to investigate undersaturated systems, the real world traffic volumes for the PM peak period were reduced such that all movements considered had saturation degrees ranging between 0.7 and 0.98 . The resulting system is referred to as System C in this study. To investigate the effects of link lengths on the results, a new network was generated by reducing the link lengths to homogeneous link lengths. We call this System D. The results are shown in Figure 43.

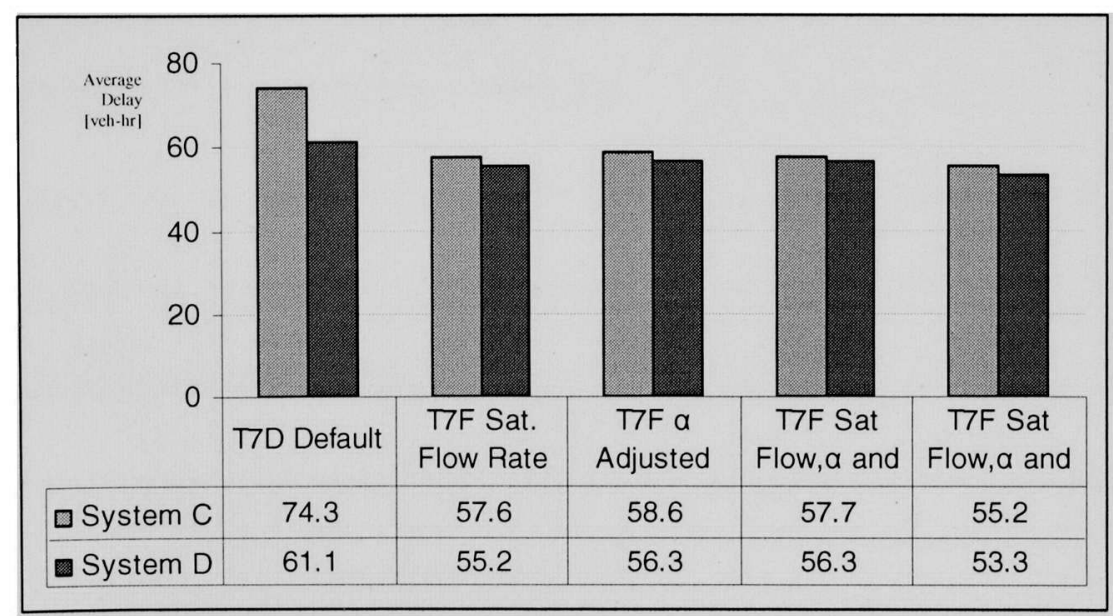

Figure 43: CORSIM Delay Estimation Different Adjustment Strategies in TRANSYT-7F 
Figure 43 shows the reduction in the system-wide average delay as an effect of different adjustment strategies. Figure 43 indicates that TRANSYT-7F was able to achieve a better solution without adjustment for links with shorter link lengths. For Systems C and D, it appears that the highest improvements in delay were obtained due to the adjustment of the saturation flow rate. Additional improvement was observed due to an adjustment of speed to 0.8 of that of CORSIM. These improvements are smaller than those observed for System A. The speed corrected version of the plan performed better in both scenarios, but the reduction was not significantly different from those obtained with other strategies.

In Systems A and B, the adjustment to the saturation flow rate was to decrease it by $16 \%$ from 2088 to 1800. In System C and D, the adjustment was increased by 4\% from 1728 to 1800 . This difference may explain why the adjustment to the saturation flow rate was more effective in Systems C and D compared to the adjustment in Systems A and B: Higher saturation flow rates allow for longer green times to accommodate those cycles with more demand or less aggressive drivers. 


\section{CONCLUSIONS AND RECOMMENDATIONS}

The following can be concluded based on the results presented in this study:

1. Longer link lengths resulted in higher delays for the same conditions due to the platoon dispersion that resulted in more vehicles arriving on red at the downstream intersection.

2. The longer the link length was, the higher the inconsistency between the CORSIM and TRANSYT-7F assessments of delays was due to the difference in platoon dispersion and the cruise speed assessments of the two models.

3. Adjusting the values of the calibration parameters of TRANSYT-7F had significant effects in improving the quality of the optimized signal plans in TRANSYT-7F as assessed by CORSIM.

4. The platoons generated using the default parameters in TRANSYT-7F were more dispersed than were the CORSIM platoons. This was particularly true for long links. In addition, more vehicles appeared to arrive at the latter stages of the green in CORSIM compared to TRANSYT-7F.

5. It appears that using a lower value of the platoon dispersion factor in TRANSYT$7 \mathrm{~F}$, based on Equations (4), (5), and (6), produced better timing plans when assessed by CORSIM. The default value for this factor in TRANSYT-7F may need to be reduced based on the microscopic simulation both in this study and in a previous study by Yu and Van Aerde (12). 
6. Reducing the cruise speed in the TRANSYT-7F model to $80 \%$ of the average cruise speed value obtained from CORSIM produced better optimized plans from TRANSYT-7F according to CORSIM. This appears to be due mainly to the ability of these plans to accommodate cycles with slower leading vehicles in the platoon.

7. Adjusting the saturation flow rate in TRANSYT-7F to make it consistent with the time headways in CORSIM may result in better optimized plans according to CORSIM.

8. Correcting the speed in TRANSYT-7F has been proven to provide the appropriate offset value when the coded speed in TRANSYT-7F is $80 \%$ of the cruise speed as assessed by CORSIM. TRANSYT-7F appeared to be able to provide almost the same offset observed form CORSIM when this adjustment was made.

9. It appears that adjusting saturation flow rate, the coded speed, and the platoon dispersion factor provided better plans as assessed by CORSIM. It is recommended that the effects of adjusting the parameters of additional macroscopic simulation models, such as shared lanes and permitted left turn movement models, be investigated. In addition, it is recommended that an additional investigation be made to assess the effectiveness of adjusting the parameters of the oversaturated optimization and simulation models, introduced during the 1990s, in TRANSYT -7F. This assessment should be made based on the produced timing plans as assessed by CORSIM. 


\section{LIST OF REFERENCES}

1. Hale, D. Network Study Tool TRANSYT-7F, United States Version T7FIO. McTrans Center, University of Florida, Gainesville, FL, 2005.

2. Fambro, D.B., Chaudhary, N.A., Messer, C.J., and Garza, R.U. A Report on the User's Manual for the Microcomputer Version of PASSER III-88: Final Report. Report No. FHWA/TX-88/478-1. Texas Transportation Institute, Texas A\&M University, College Station, TX, September 1988.

3. Traffic Synchro Software. Synchro v2.0 Users's Manual and Professional Features. 1995.

4. Federal Highway Administration. CORSIM User's Manual. U.S. Department of Transportation, Office of Safety and Traffic Operations R\&D, Intelligent Systems and Technology, 1997.

5. Denney, R.W.J. "Traffic Platoon Dispersion Modeling." Journal of Transportation Engineering, 115(2), pp.193-207, 1989.

6. Highway Capacity Manual 2000, Transportation Research Board, 2000.

7. Yan, J. "Using TRAF-NETSIM to Evaluate Signal Optimization Programs for Arterial Streets." $67^{\text {th }}$ Annual Meeting Compendium of Technical Papers, Boston, MA, 1997.

8. Park, B., Ruphail, N.M., Hochanadel, J.P., and Sacks, J. "Evaluating Reliability of TRANSYT-7F Optimization Schemes." Journal of Transportation Engineering, 127(4), pp.319-326, 2001.

9. Ruphail, N., Park, B., and Sacks, J. "Direct Signal Timing Optimization: Strategy Development and Results." 2006.

10. Stevanovic, A. and Martin, P.T. "Evaluating the Reliability of Macroscopically Optimized Timing Plans Through Microsimulation." TRB 2006 Annual Meeting, January 2006.

11. Seddom, P.A. "Another Look at Platoon Dispersion Along a Major Road in Sheffield." Traffic Engineering and Control, 13(10), 1972, pp. 442-444.

12. Yu, L. and Van Aerde, M. "Implementing TRANSYT's Macroscopic Platoon Dispersion in Microsimulation Models." 1995. Transportation Research Board Annual Meeting, paper\# 950803. Washington, DC. 1995. 